\title{
Mytilus galloprovincialis and M. edulis on the coasts of the Iberian Peninsula
}

\author{
A. Sanjuan*, C. Zapata, G. Alvarez \\ Departamento de Biología Fundamental, Facultad de Biología, Universidad de Santiago de Compostela, E-15706 Santiago \\ de Compostela, Spain
}

\begin{abstract}
Mytilus edulis L. and $M$. galloprovincialis Lmk. are 2 forms of mussels that live on the European coasts, and where they coexist, hybridize in varying proportions. While the Atlantic coast of the Iberian Peninsula is a key area for understanding the distribution of the genus Mytilus in Europe, the available evidence on the distribution from this area is scarce and contradictory. Five partially diagnostic allozyme loci (aminopeptidase-1, esterase-D, leucine aminopeptidase-1, mannose phosphate isomerase and octopine dehydrogenase) and the best diagnostic morphological characters (length of the anterior adductor muscle scar and internal radius of the hinge plate and shell length and height) between $M$. edulis and $M$. galloprovincialis were investigated in 37 and 39 samples respectively taken from the Iberian coasts. Concordance between morphological variation and enzyme polymorphisms is virtually complete and indicates that $M$. galloprovincialis occurs all along the Iberian Peninsula (Mediterranean, Lusitanian, Galician Rías and Cantabrian coasts). M. galloprovincialis follows, therefore, a continuous distribution all along the Atlantic coast of Europe, from the Mediterranean Sea to the English Channel and the British Isles. Moreover, M. galloprovincialis exhibits an abrupt discontinuity in allozyme frequencies of about $40 \%$ for Odh 100 and $25 \%$ for Ap- $1 \cdot 100$ in the southeastern Mediterranean Iberian coasts. This strong genetic differentiation appears to be associated with an oceanographic current barrier (the Almeria-Oran front). On the other hand, evidence for the presence of $M$. edulis and hybrids of $M$. edulis and $M$. galloprovincialis was obtained only on the Atlantic coast near the Hispano-French frontier. Therefore, it seems that the meridional limit of the geographical distribution of $M$. edulis in Europe, and the concomitant limit of the hybrid zone, are between the mouths of the Bidasoa and Nervion rivers in Spain. These results clarify some evolutionary aspects of $M$. edulis and $M$. galloprovincialis.
\end{abstract}

KEY WORDS: Biogeography - Iberian Peninsula $\cdot$ Mytilus - Population genetics - Isoenzyme polymorphisms

\section{INTRODUCTION}

Three forms of mussels, Mytilus edulis L. (blue mussel), M. galloprovincialis Lmk. (Mediterranean mussel) and $M$. trossulus Gould, have been distinguished on the European coasts basically on grounds of morphology and allozymes (see Skibinski et al. 1983, Gosling 1984, 1992a, b, Koehn 1991, Gardner 1992, Seed 1992). Mytilus edulis has been recognized with confidence along the Atlantic coast of Europe, from the northern

- Present address: Laboratorio de Xenética, Facultade de Ciencias, Apdo 874, Universidade de Vigo, E-36200 Vigo, Spain
White Sea (McDonald et al. 1990) through the western European coast including Iceland and the British Isles (Seed 1976, Skibinski et al 1983, Varvio et al 1988, McDonald et al. 1991) as far south as the Atlantic French coast (Seed 1972, 1976, Verduin 1979, Coustau et al. 1991, McDonald et al. 1991). M. galloprovincialis has been unambiguously identified on the Mediterranean coast, on the British Isles and on the Atlantic coast of France (Seed 1972, 1976, Verduin 1979, Skibinski et al. 1983, Coustau et al. 1991, McDonald et al. 1991; see Gosling 1984). A third form, M. trossulus, has been identified on the basis of electrophoretic data and, in Europe, seems confined to the Baltic Sea (Bulnheim \& Gosling 1988, Varvio et al. 1988, Johan- 
nesson et al. 1990). M. edulis and $M$. galloprovincialis coexist in varying proportions in some areas of the British Isles and Atlantic coast of France, where they hybridize as shown by allozyme and mitochondrial DNA data (Ahmad \& Beardmore 1976, Skibinski et al. 1978, 1983, Skibinski 1985, Edwards \& Skibinski 1987. Beaumont et al. 1989, Coustau et al. 1991, Hoeh et al. 1991, McDonald et al. 1991; for reviews see Koehn 1991, Gardner 1992, Gosling 1992a, b, Seed 1992). The proportion of the 2 mussel forms and the degree of hybridization and introgression depend on the sample site and local conditions such as wave exposure and attachment height (see Gosling 1992b).

Current knowledge of the distribution of the different forms of mussels in the European coasts is incomplete, particularly with respect to the Iberian Peninsula coasts. The Atlantic coast of the Iberian Peninsula is a key area for understanding the distribution of the genus Mytilus on the Atlantic coasts of Europe for 2 important reasons. First, the occurrence of Mytilus galloprovincialis in the British Isles and on the Atlantic coasts of France can be understood differently according to whether the distribution of $M$. galloprovincialis is continuous or discontinuous from the Mediterranean Sea to those coasts. Second, study of the mussels on the Atlantic Iberian coast will establish the southern extent of the distribution of $M$. edulis in Europe and the range of the hybrid zone of $M$. edulis and $M$. galloprovincialis (Koehn 1991, Gardner 1992, Gosling 1992b, Seed 1992). In spite of this, the distribution of Mytilus spp. on the Atlantic coast of the Iberian Peninsula has not been investigated thoroughly and there are scarce and contradictory data. One opinion is that $M$. edulis extends over the whole of the Atlantic coast of Europe as far south as northern Africa; another opinion is that $M$. galloprovincialis is the predominant mussel on the Atlantic coasts of the Iberian Peninsula (Lubet 1973. 1976. Seed 1976, 1978, Suchanek 1985). To date, studies designed to extensively and reliably analyze mussel populations have been conducted only in northwestern Iberia, and they indicate the occurrence of M. galloprovincialis (Sanjuan et al. 1986, 1990). Two macrogeographical surveys with allozyme characters including other locations on the Iberian coasts indicate the occurrence of $M$. galloprovincialis (in Albufeira; Bulnheim \& Gosling 1988) and hybrids of the 2 mussel forms (in Parede; McDonald et al. 1991). The other references about the distribution of Mytilus in the Atlantic Iberian coasts are based on partial data with limited numbers of samples or individuals or use unreliable discriminant characters (for reviews see Sanjuan et al. 1986, Sanjuan 1992, and references therein). To date, no clear conclusion has been reached with regard to the identity of the mussels living on these coasts. The main aim of this work is, therefore, to identify the form or forms of mussels that live on the coasts of Iberian Peninsula. For this purpose diagnostic allozyme loci and morphological characters have been studied in mussel populations from the Iberian Peninsula.

\section{MATERIALS AND METHODS}

Populations sampled. Mussel populations were sampled along the Atlantic and Mediterranean coasts of the Iberian Peninsula from 1985 to 1988 (Table 1, Fig. 1). Most samples were collected as close as possible to the low level of the mussel bed, but some came from commercial cultures and one came from submerged rocks at $9 \mathrm{~m}$ depth. For comparative purposes commercial and wild pure populations of Mytilus edulis from the northwestern Atlantic coasts of Europe were also studied (Table 1). Mussels were brought alive to the laboratory as quickly as possible, where they were dissected. The digestive gland was removed from each individual and frozen at $-70^{\circ} \mathrm{C}$ until prepared for allozyme analysis.

Length of anterior adductor muscle scar and internal radius of the hinge plate, the best diagnostic conchological characters between Mytilus edulis and M. galloprovincialis (Lewis \& Seed 1969, Seed 1974, 1978, Verduin 1979, Sanjuan et al. 1986, 1990, Beaumont et al. 1989, Sanjuan 1992), and length and height of the shells were examined in 39 mussel samples. The length of the anterior adductor muscle scar was measured in dry shells and the internal radius of the hinge

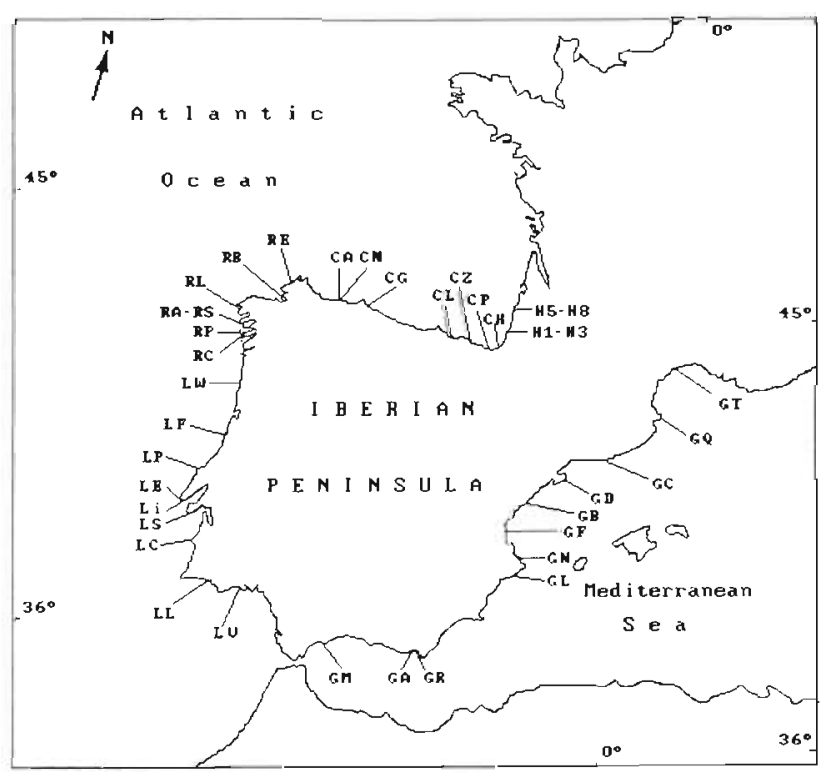

Fig. 1. Collection sites of Mytilus populations on the Iberian Peninsula coasts: Mediterranean (G_), Lusitanian (L_); Galician Rías ( $R_{-}$); Hispano-Cantabrian $\left(C_{-}\right)$and French-Cantabrian (H_) populations. Population codes as in Table 1 
Table 1. Mytilus spp. Iberian Peninsula mussel populations and typical $M$. edulis (E_) from northwestern Europe for comparison. Code is a population code; $\mathrm{N}$ : number of individuals scored; Morph.: samples analyzed for morphological characters Loci: samples analyzed for allozyme loci

\begin{tabular}{|c|c|c|c|c|c|}
\hline Populations & Code & Location & $N$ & Morph. & Loci \\
\hline \multicolumn{6}{|c|}{ Mediterranean coasts (M. galloprovincialis) } \\
\hline Thau & GT & Basin de Thau (culture) & 41 & & + \\
\hline Cadaques & $\mathrm{GQ}$ & Cadaques & 74 & & + \\
\hline Cambrils & $\mathrm{GC}$ & Cambrils & 65 & + & + \\
\hline Delta & GD & Delta Ebro (culture) & 89 & + & + \\
\hline Benicasim & GB & Benicasim & 100 & + & \\
\hline Cullera & GF & Faro de Cullera & 74 & + & \\
\hline Denia & GN & Denia & 50 & + & \\
\hline Altea & GL & Altea & 67 & + & \\
\hline Almería-1 & GR & Almería (east beach) & 93 & + & \\
\hline Almería-2 & $\mathrm{GA}$ & Almeria (west beach) & 82 & & + \\
\hline Marbella & GM & Marbella (groynes) & 78 & + & + \\
\hline \multicolumn{6}{|l|}{ Lusitanian coasts } \\
\hline Vilamoura & LV & Vilamoura & 105 & + & + \\
\hline Lagos & LL & Lagos & 110 & + & + \\
\hline Cabo Sines & $\mathrm{LC}$ & Cabo Sines & 65 & + & + \\
\hline Sezimbra & LS & Sezimbra & 110 & + & + \\
\hline Lisboa & $\mathrm{Li}$ & Lisboa (harbour) & 74 & + & + \\
\hline Estoril & LE & Estoril & 110 & + & + \\
\hline Peniche & $\mathrm{LP}$ & Peniche (breakwater) & 100 & & + \\
\hline Figueira & $\mathrm{LF}$ & Figueira da Foz (harbour) & 111 & & + \\
\hline Varzim & $\mathrm{LW}$ & Povoa de Varzim & 110 & & + \\
\hline \multicolumn{6}{|l|}{ Galician Rías } \\
\hline Pontevedra-5 & $\mathrm{RC}$ & Aldán (culture) & 137 & + & + \\
\hline Pontevedra-7 & $R P$ & Pontevedra & 110 & + & + \\
\hline Arousa-3 & RA & Carril & 112 & + & + \\
\hline Arousa-4 & RS & Illa da Rua (submerged) & 107 & & + \\
\hline Laxe-3 & RL & Laxe (harbour) & 100 & + & + \\
\hline Ares-5 & RB & Sada & 125 & + & + \\
\hline Cedeira & RE & Cedeira & 100 & + & + \\
\hline \multicolumn{6}{|l|}{ Bay of Biscay } \\
\hline Navia-1 & CA & Navia & 97 & + & \\
\hline Navia-2 & $\mathrm{CN}$ & Navia & 36 & + & + \\
\hline Gijón & $\mathrm{CG}$ & Gijón & 110 & + & + \\
\hline La Arena & $\mathrm{CL}$ & La Arena (beach) & 161 & & + \\
\hline Zierbena & $\mathrm{CZ}$ & Zierbena (harbour) & 121 & + & + \\
\hline Pasaia-B & $C P$ & San Pedro de Pasaia & 76 & & + \\
\hline Hondarribia & $\mathrm{CH}$ & Hondarribia & 120 & + & + \\
\hline Adour-DA & $\mathrm{H} 1$ & Barre de L'Adour (breakwater) & 120 & + & + \\
\hline Adour-DB & $\mathrm{H} 2$ & Barre de L'Adour (breakwater) & 30 & + & + \\
\hline Adour-CC & $\mathrm{H} 3$ & Barre de L'Adour (channel) & 109 & + & + \\
\hline Capbreton-CA & H5 & Capbreton (channel) & 110 & & + \\
\hline Capbreton-CB & H6 & Capbreton (channel) & 226 & + & + \\
\hline Capbreton-FB & $\mathrm{H7}$ & Capbreton (groynes) & 110 & + & + \\
\hline Capbreton-FA & $\mathrm{H} 8$ & Capbreton (groynes) & 23 & + & \\
\hline \multicolumn{6}{|c|}{ Northwestern Europe (M. edulis) } \\
\hline France-R & ER & Riec-sur-Mer (culture) & 70 & + & + \\
\hline France-D & ED & Dieppe & 29 & + & \\
\hline Belgium & $\mathrm{EO}$ & Oostende & 46 & + & \\
\hline Holland-1 & $\mathrm{EC}$ & Holland (culture) & 102 & + & + \\
\hline Holland-2 & EN & Holland (culture) & 57 & + & + \\
\hline Wales & EA & Anglesey & 17 & + & \\
\hline Denmark-1 & $\mathrm{EH}$ & Helsingør & 43 & + & \\
\hline Denmark-2 & $E B$ & Blåvands & 60 & + & \\
\hline
\end{tabular}

plate was measured according to Verduin (1979). Because there are high correlations between some characters and shell length, the 3 original variables were transformed by dividing the measures by the shell length (Seed 1978, 1992, Beaumont et al. 1989).
Electrophoresis. Five partially diagnostic allozyme loci found to be informative for distinguishing between Mytilus edulis and M. galloprovincialis (see Ahmad \& Beardmore 1976, Skibinski et al. 1978, 1980, 1983, Grant \& Cherry 1985, Beaumont et al. 
1989) were investigated by starch-gel electrophoresis in 37 samples. The digestive glands were homogenized in an equal volume of $0.01 \mathrm{M}$ dithiothreitol solution. All the homogenized tissue samples were centrifuged at $9000 \mathrm{rpm}(8000 \times g)$ for $7 \mathrm{~min}$ and the supernatant was used as the enzyme source. Electrophoresis was carried out on 10 to $12 \%$ horizontal starch gels at $4^{\circ} \mathrm{C}$ using standard techniques (Harris \& Hopkinson 1976, Pasteur et al. 1987). Enzymatic systems were aminopeptidase-1 $\left(A p-1^{*}\right)$, esterase- $D$ $\left(\right.$ Est- $\left.D^{*}\right)$, leucine aminopeptidase-1 (Lap-1*), octopine dehydrogenase $\left(\mathrm{Odh}^{\circ}\right)$ and mannose phosphate isomerase $\left(\mathrm{Mpi}^{*}\right)$. Electrophoretic procedures and terminology were basically those described in Ahmad et al. (1977) and Skibinski et al. (1980) for Ap-1*, Est- $D^{*}$, and $\operatorname{Lap}-1^{\circ}$, Grant \& Cherry (1985) for Odh and Sanjuan et al. (1990) for Mpi*

Data analysis. The genetic structure within and among populations was analyzed using Wright's $F$ statistics $\left(F_{I S}, F_{S T}\right.$ and $\left.F_{I T}\right)$. F-statistics were calculated by the method of Nei (1977, 1986; see also Nei \& Chesser 1983). Estimation of $F_{I S}$ index in each mussel population was carried out by a statistic developed by Robertson \& Hill (1984), because it is unbiased for $F=0$ and a significance test for $F=0$ exists which has a higher statistical power than the usual chi-square test. The $F_{I S}$ statistic was used to measure the deviation of genotype frequencies from Hardy-Weinberg proportions; positive values indicated a deficit of heterozygotes and negative values an excess. Estimation of Nei's genetic distance (Nei 1972) was carried out among pairs of populations.

Multivariate analyses were used to provide a lowdimensional representation of the data and the clearest possible description of the relations between the populations (Pimentel 1979, Reyment et al. 1984, James \& McCulloch 1990). A hierarchical cluster analysis using the unweighted pair-group method with arithmetic averaging (UPGMA) was applied to the matrix of pairwise genetic distances (Sneath \& Sokal 1973, Dunn \& Everitt 1982). The cophenetic correlation was calculated and was used as a measure of the goodness of fit of the dendrogram to the original matrix of distances (Sneath \& Sokal 1973). The samples were also ordinated with non-metric multidimensional scaling of the distance matrices (Dunn \& Everitt 1982, Reyment et al. 1984). This method attempts to arrange the samples along few dimensions, so that similar samples are together and dissimilar samples are far apart. The stress, which measures the monoticity of the distances in the final configuration with the observed distances, was calculated. Low values of stress indicate a high degree of concordance of the rank orderings of new configuration and original distances. A minimum length spanning tree from the distance matrices was also computed and superimposed on the ordination diagram to graphically detect local distortions (Dunn \& Everitt 1982, Rohlf 1990).

A composite genetic or hybrid index score for each individual based on its genotype at the 4 best diagnostic loci surveyed (Est- $D^{*}, L a p-1^{*}, O d h^{*}$ and $\left.M p i^{*}\right)$, was calculated. A score of -1 was assigned to the typical alleles of Mytilus galloprovincialis or diagnostic allozymes with a high frequency in $M$. galloprovincialis control populations (' $G$ ' compound allele), a score of +1 was assigned to the typical alleles of $M$. edulis ( $\mathrm{E}^{\prime}$ compound allele), and a value of 0 was assigned to other alleles. The composite index score of an individual represented the sum of its scores over all 4 loci. So, the score of an individual could range from -8 ( $M$. galloprovincialis) to +8 ( $M$. edulis).

Multivariate analyses were carried out using the NTSYS-pc computer program (Rohlf 1990), and most genetic analyses were performed with GENET2 (Quesada et al. 1992). Conventional statistical calculations were carried out with the SPSS/PC package (Nie et al. 1975).

\section{RESULTS}

Allozyme frequencies at the 5 loci $A p-1^{\circ}$, Est- $D^{*}$, Lap-1 ${ }^{*}, M p i^{*}$ and $O d h^{*}$ for the 37 samples are shown in Tables 2 to 6 . These allozyme loci are partially diagnostic for Mytilus edulis and M. galloprovincialis (see Skibinski et al. 1983, McDonald et al. 1991, Gardner 1992, Gosling 1992a, b, Seed 1992). The allele frequency distributions at the 5 loci in mussel populations from northwestern Europe coast $\left(E_{-}\right)$and from the Mediterranean coast ( $G_{-}$), were used as control samples of $M$. edulis and $M$. galloprovincialis respectively and agreed with allele frequencies observed by other authors for both mussel forms (see Gardner 1992, Gosling 1992b). Est- $\mathrm{D}^{*}$ and $\mathrm{Mpi}^{*}$ loci presented a single allozyme (Est-D*90 and $\mathrm{Mpi}$ (100), and Lap-1* and $O d h^{*}$ a pair of pooled allozymes (Lap-1 104 and Lap-1 108 , and $\mathrm{Odh}^{*} 100$ and $\mathrm{Odh} \cdot 129$ ) at high frequency in $M$. galloprovincialis collections (about 70 to $97 \%$ ) and at low frequency in $M$. edulis ( 1 to $13 \%$ ). In contrast, other allozymes at each locus, such as Est-D*100, Mpi*200, Odh·115, and Lap-1·96 and Lap-1 100 pooled, were at low frequency ( 1 to $29 \%$ ) in M. galloprovincialis and at high frequency (87 to $97 \%$ ) in $M$. edulis. Ap- $1^{*}$ had a weaker discriminatory power.

Data of the allozyme frequencies for all populations are summarized by cluster and ordination analyses based on Nei's (1972) genetic distance. The UPGMA dendrogram (Fig. 2) shows that the deepest dichotomy (distance $=1.08$ ) occurred between a small group, which includes the control Mytilus edulis ( $\left.\mathrm{E}_{-}\right)$and 
Table 2. Mytilus spp. Allele frequencies at the Ap- $1^{\bullet}$ locus for Iberian Peninsula mussel populations and for typical $M$. edulis

\begin{tabular}{|c|c|c|c|c|c|c|c|}
\hline Population & 86 & 93 & 100 & 108 & 114 & 122 & 128 \\
\hline \multicolumn{8}{|c|}{ Mediterranean coasts (M. galloprovincialis) } \\
\hline GT & 0 & 0.012 & 0.195 & 0.280 & 0.378 & 0.122 & 0.012 \\
\hline $\mathrm{GQ}$ & 0 & 0.007 & 0.164 & 0.452 & 0.233 & 0.137 & 0.007 \\
\hline $\mathrm{GC}$ & 0 & 0.008 & 0.162 & 0.400 & 0.285 & 0.123 & 0.023 \\
\hline GD & 0 & 0.007 & 0.184 & 0.390 & 0.250 & 0.162 & 0.007 \\
\hline GA & 0 & 0.024 & 0.421 & 0.341 & 0.177 & 0.037 & 0 \\
\hline GM & 0 & 0.045 & 0.344 & 0.390 & 0.175 & 0.026 & 0.019 \\
\hline \multicolumn{8}{|c|}{ Lusitanian coasts } \\
\hline LV & 0 & 0.013 & 0.475 & 0.275 & 0.206 & 0.031 & 0 \\
\hline LL & 0 & 0.014 & 0.423 & 0.355 & 0.150 & 0.050 & 0.009 \\
\hline LC & 0 & 0 & 0.400 & 0.369 & 0.177 & 0.054 & 0 \\
\hline LS & 0 & 0.009 & 0.436 & 0.327 & 0.191 & 0.036 & 0 \\
\hline $\mathrm{Li}$ & 0 & 0.007 & 0.493 & 0.314 & 0.150 & 0.036 & 0 \\
\hline LE & 0 & 0.023 & 0.393 & 0.369 & 0.192 & 0.023 & 0 \\
\hline LP & 0 & 0.010 & 0.400 & 0.355 & 0.185 & 0.050 & 0 \\
\hline LF & 0 & 0.009 & 0.450 & 0.351 & 0.144 & 0.041 & 0.005 \\
\hline $\mathrm{LW}$ & 0 & 0.014 & 0.418 & 0.327 & 0.209 & 0.032 & 0 \\
\hline \multicolumn{8}{|c|}{ Galician Rías } \\
\hline $\mathrm{RC}$ & 0.011 & 0.011 & 0.407 & 0.332 & 0.200 & 0.032 & 0.007 \\
\hline $\mathrm{RP}$ & 0.006 & 0 & 0.392 & 0.380 & 0.169 & 0.054 & 0 \\
\hline RA & 0 & 0,005 & 0.429 & 0.344 & 0.160 & 0.057 & 0.005 \\
\hline RS & 0 & 0.005 & 0.446 & 0.322 & 0.188 & 0.040 & 0 \\
\hline RL & 0.005 & 0.014 & 0.389 & 0.361 & 0.144 & 0.083 & 0.005 \\
\hline $\mathrm{RB}$ & 0.004 & 0.012 & 0.455 & 0.361 & 0.127 & 0.041 & 0 \\
\hline RE & 0 & 0.010 & 0.485 & 0.285 & 0.165 & 0.050 & 0.005 \\
\hline \multicolumn{8}{|c|}{ Bay of Biscay } \\
\hline $\mathrm{CN}$ & 0 & 0.014 & 0.361 & 0.306 & 0.250 & 0.069 & 0 \\
\hline $\mathrm{CG}$ & 0 & 0.005 & 0.464 & 0.318 & 0.191 & 0.023 & 0 \\
\hline CL & 0.003 & 0.006 & 0.440 & 0.326 & 0.168 & 0.047 & 0.009 \\
\hline $\mathrm{CZ}$ & 0.004 & 0.004 & 0.425 & 0.325 & 0.196 & 0.038 & 0.008 \\
\hline $\mathrm{CP}$ & 0 & 0.007 & 0.375 & 0.342 & 0.197 & 0.079 & 0 \\
\hline $\mathrm{CH}$ & 0 & 0.004 & 0.432 & 0.297 & 0.212 & 0.051 & 0.004 \\
\hline $\mathrm{H} 1$ & 0 & 0.025 & 0.500 & 0.303 & 0.143 & 0.025 & 0.004 \\
\hline $\mathrm{H} 2$ & 0 & 0 & 0.433 & 0.283 & 0.267 & 0.017 & 0 \\
\hline $\mathrm{H} 3$ & 0 & 0 & 0.532 & 0.255 & 0.167 & 0.046 & 0 \\
\hline H5 & 0.004 & 0.017 & 0.558 & 0.262 & 0.146 & 0.008 & 0.004 \\
\hline $\mathrm{H} 6$ & 0.004 & 0.004 & 0.681 & 0.201 & 0.073 & 0.029 & 0.007 \\
\hline $\mathrm{H} 7$ & 0 & 0.014 & 0.436 & 0.332 & 0.182 & 0.032 & 0.005 \\
\hline \multicolumn{8}{|c|}{ Northwestern Europe ( $M$. edulis) } \\
\hline ER & 0.007 & 0.007 & 0.799 & 0.157 & 0.030 & 0 & 0 \\
\hline $\mathrm{EC}$ & 0 & 0.021 & 0.819 & 0.111 & 0.042 & 0.007 & 0 \\
\hline EN & 0 & 0 & 0.850 & 0.140 & 0.010 & 0 & 0 \\
\hline
\end{tabular}

some French-Cantabrian populations (H5, H6), and a large group, which includes the control Mediterranean M. galloprovincialis populations $\left(\mathrm{G}_{-}\right)$and the remaining populations (Atlantic Iberian populations and the other French-Cantabrian samples H1, H2, H3 and H7). For the $M$. galloprovincialis cluster, another dichotomy occurred at a distance of 0.08 with a small group of 4 Mediterranean samples from the northeastern Iberian coast (GT, GQ, GC, and GD) and a larger group, including 2 Mediterranean populations from southern Iberian coasts (GA, GM) and the Atlantic samples $\left(L_{-}, R_{-}, C_{-}\right.$, and $\left.H 1, H 2, H 3, H 7\right)$. Some samples from Capbreton (H5 and H6) were grouped with pure $M$. edulis populations and the other $(\mathrm{H} 7)$ with the $M$. gal- loprovincialis cluster. The plots of 2-dimensional projections resulting from a non-metric multidimensional analysis based on Nei's (1972) genetic distance upon the 5 loci and each individual loci clarified the relationship between the populations (Fig. 3). The overall pattern based upon the 5 loci shows the extreme separation of the $3 \mathrm{M}$. edulis control samples $\left(\mathrm{E}_{-}\right)$and the Mediterranean $M$. galloprovincialis control samples (G_). Moreover, the 4 Mediterranean samples from the northeastern Iberian coast (GT, GQ, GC, and GD) were separated from the large group, including the 2 Mediterranean populations from the south Iberian littoral (GA, GM) and most of the Atlantic Iberian populations (Lusitanian L_, Galician R_ and Hispano-Cantabrian 
Table 3. Mytilus spp. Allele frequencies at the Est- $D^{*}$ locus for Iberian Peninsula mussel populations and for typical $M$. edulis

\begin{tabular}{|c|c|c|c|c|c|c|c|c|c|}
\hline Population & 76 & 82 & 87 & 90 & 93 & 100 & 103 & 107 & 110 \\
\hline \multicolumn{10}{|c|}{ Mediterranean coasts (M. galloprovincialis) } \\
\hline GT & 0.049 & 0.012 & 0 & 0.878 & 0 & 0.061 & 0 & 0 & 0 \\
\hline$G Q$ & 0.034 & 0.007 & 0 & 0.939 & 0 & 0.020 & 0 & 0 & 0 \\
\hline $\mathrm{GC}$ & 0.031 & 0.038 & 0 & 0.900 & 0 & 0.031 & 0 & 0 & 0 \\
\hline $\mathrm{GD}$ & 0.007 & 0 & 0 & 0.971 & 0 & 0.022 & 0 & 0 & 0 \\
\hline $\mathrm{GA}$ & 0 & 0.037 & 0 & 0.939 & 0.012 & 0.012 & 0 & 0 & 0 \\
\hline GM & 0.006 & 0.058 & 0 & 0.909 & 0 & 0.013 & 0.013 & 0 & 0 \\
\hline \multicolumn{10}{|c|}{ Lusitanian coasts } \\
\hline LV & 0 & 0.031 & 0 & 0.931 & 0.006 & 0.031 & 0 & 0 & 0 \\
\hline LL & 0 & 0.051 & 0 & 0.866 & 0.032 & 0.046 & 0.005 & 0 & 0 \\
\hline LC & 0 & 0.023 & 0 & 0.883 & 0.008 & 0.086 & 0 & 0 & 0 \\
\hline LS & 0 & 0.055 & 0 & 0.864 & 0.014 & 0.068 & 0 & 0 & 0 \\
\hline $\mathrm{Li}$ & 0.007 & 0.058 & 0 & 0.870 & 0 & 0.065 & 0 & 0 & 0 \\
\hline LE & 0.005 & 0.037 & 0 & 0.911 & 0.009 & 0.037 & 0 & 0 & 0 \\
\hline LP & 0.015 & 0.030 & 0.010 & 0.890 & 0 & 0.055 & 0 & 0 & 0 \\
\hline LF & 0 & 0.059 & 0 & 0.891 & 0.009 & 0.041 & 0 & 0 & 0 \\
\hline LW & 0.005 & 0.048 & 0 & 0.936 & 0.011 & 0 & 0 & 0 & 0 \\
\hline \multicolumn{10}{|c|}{ Galician Rias } \\
\hline $\mathrm{RC}$ & 0.004 & 0.040 & 0 & 0.885 & 0 & 0.072 & 0 & 0 & 0 \\
\hline $\mathrm{RP}$ & 0 & 0.026 & 0 & 0.953 & 0.005 & 0.016 & 0 & 0 & 0 \\
\hline RA & 0.005 & 0.029 & 0 & 0.880 & 0.005 & 0.063 & 0.005 & 0.010 & 0.005 \\
\hline RS & 0.009 & 0.014 & 0 & 0.907 & 0.019 & 0.051 & 0 & 0 & 0 \\
\hline $\mathrm{RL}$ & 0 & 0.037 & 0 & 0.903 & 0.005 & 0.056 & 0 & 0 & 0 \\
\hline RB & 0 & 0.039 & 0 & 0.917 & 0.020 & 0.020 & 0 & 0.005 & 0 \\
\hline $\mathrm{RE}$ & 0 & 0.040 & 0 & 0.935 & 0 & 0.025 & 0 & 0 & 0 \\
\hline \multicolumn{10}{|c|}{ Bay of Biscay } \\
\hline CN & 0.014 & 0.014 & 0 & 0.917 & 0 & 0.056 & 0 & 0 & 0 \\
\hline $\mathrm{CG}$ & 0.005 & 0.050 & 0 & 0.901 & 0.005 & 0.036 & 0.005 & 0 & 0 \\
\hline CL & 0.003 & 0.034 & 0 & 0.910 & 0.003 & 0.047 & 0.003 & 0 & 0 \\
\hline $\mathrm{CZ}$ & 0.004 & 0.033 & 0 & 0.896 & 0 & 0.063 & 0.004 & 0 & 0 \\
\hline $\mathrm{CP}$ & 0 & 0.052 & 0 & 0.870 & 0 & 0.078 & 0 & 0 & 0 \\
\hline $\mathrm{CH}$ & 0 & 0.029 & 0 & 0.917 & 0 & 0.050 & 0 & 0.004 & 0 \\
\hline $\mathrm{H} 1$ & 0 & 0.038 & 0 & 0.811 & 0 & 0.139 & 0.008 & 0.004 & 0 \\
\hline $\mathrm{H} 2$ & 0 & 0.033 & 0 & 0.800 & 0 & 0.150 & 0.017 & 0 & 0 \\
\hline H3 & 0.009 & 0.018 & 0 & 0.786 & 0 & 0.182 & 0.005 & 0 & 0 \\
\hline H5 & 0.004 & 0.016 & 0 & 0.423 & 0 & 0.537 & 0.004 & 0.016 & 0 \\
\hline H6 & 0.002 & 0.013 & 0 & 0.271 & 0.002 & 0.687 & 0 & 0.013 & 0.011 \\
\hline $\mathrm{H} 7$ & 0 & 0.036 & 0 & 0.655 & 0.018 & 0.286 & 0 & 0.005 & 0 \\
\hline \multicolumn{10}{|c|}{ Northwestern Europe ( $M$. edulis) } \\
\hline ER & 0 & 0 & 0 & 0.014 & 0 & 0.943 & 0 & 0.007 & 0.036 \\
\hline $\mathrm{EC}$ & 0 & 0 & 0 & 0.022 & 0 & 0.941 & 0 & 0.007 & 0.029 \\
\hline EN & 0 & 0 & 0 & 0.050 & 0 & 0.910 & 0 & 0.020 & 0.020 \\
\hline
\end{tabular}

C_samples). The French-Cantabrian populations ( $\left.\mathrm{H}_{-}\right)$, except $\mathrm{H} 2$, were intermediately situated between the $M$. edulis cluster and the $M$. galloprovincialis group as shown by the minimum spanning tree diagram superimposed on the ordination analysis (Fig. 3; 5 loci). Some samples from the same locality of Capbreton as H5 and H6 (Capbreton channel) were close to the $M$. edulis cluster whereas H7 (Capbreton groynes) was near to the $M$. galloprovincialis group. Individual loci contributed differentially to the overall pattern of genetic divergence. Est- $D^{*}$, Lap-1 ${ }^{*}$, and Mpi* loci produced non-metric multidimensional patterns with 2 main groups corresponding to $M$. edulis and $M$. galloprovincialis populations, whereas $A p-1^{*}$ and $O d h^{*}$ loci gave the above 3 groups (see Fig. 3 for each locus). In all cases mussel populations from the FrenchCantabrian coasts fell between the $M$. edulis and $M$. galloprovincialis extreme clusters: H5 and H6 (Capbreton channel) were closer to the $M$. edulis cluster in all cases, whereas $\mathrm{H} 1, \mathrm{H} 2$, and $\mathrm{H} 3$ (from Barre l'Adour) and $\mathrm{H} 7$ (from Capbreton groynes) were nearest to the M. galloprovincialis cluster with a varying arrangement.

In addition, a multivariate analysis of 3 diagnostic conchological characters between Mytilus edulis and M. galloprovincialis was carried out on the mussel populations. The raw data from each population are in Sanjuan (1992). The representation of non-metric 
Table 4. Mytilus spp. Allele frequencies at the Lap-1 locus for Iberian Peninsula mussel populations and for typical $M$. edulis

\begin{tabular}{|c|c|c|c|c|c|c|c|}
\hline Population & 93 & 96 & 100 & 102 & 104 & 108 & 110 \\
\hline \multicolumn{8}{|c|}{ Mediterranean coasts (M. galloprovincialis) } \\
\hline GT & 0 & 0 & 0.049 & 0.122 & 0.390 & 0.427 & 0.012 \\
\hline $\mathrm{GQ}$ & 0.007 & 0.020 & 0.027 & 0.027 & 0.372 & 0.534 & 0.014 \\
\hline $\mathrm{GC}$ & 0 & 0 & 0.048 & 0.056 & 0.476 & 0.373 & 0.048 \\
\hline GD & 0 & 0.044 & 0.074 & 0.059 & 0.346 & 0.456 & 0.022 \\
\hline GA & 0 & 0 & 0.056 & 0.031 & 0.395 & 0.494 & 0.025 \\
\hline GM & 0 & 0 & 0.027 & 0.009 & 0.420 & 0.518 & 0.027 \\
\hline \multicolumn{8}{|c|}{ Lusitanian coasts } \\
\hline LV & 0 & 0.006 & 0.038 & 0.025 & 0.438 & 0.456 & 0.038 \\
\hline LL & 0 & 0 & 0.052 & 0.033 & 0.392 & 0.500 & 0.024 \\
\hline LC & 0 & 0.008 & 0.016 & 0.016 & 0.430 & 0.438 & 0.094 \\
\hline LS & 0.009 & 0.009 & 0.027 & 0.009 & 0.432 & 0.477 & 0.036 \\
\hline $\mathrm{Li}$ & 0 & 0 & 0.029 & 0.014 & 0.464 & 0.478 & 0.014 \\
\hline LE & 0 & 0.025 & 0.025 & 0.010 & 0.445 & 0.480 & 0.015 \\
\hline LP & 0 & 0 & 0.051 & 0.025 & 0.460 & 0.444 & 0.020 \\
\hline LF & 0 & 0.009 & 0.064 & 0.018 & 0.390 & 0.482 & 0.037 \\
\hline LW & 0 & 0.009 & 0.050 & 0.027 & 0.450 & 0.427 & 0.036 \\
\hline \multicolumn{8}{|c|}{ Galician Rías } \\
\hline $\mathrm{RC}$ & 0 & 0.011 & 0.051 & 0.011 & 0.394 & 0.504 & 0.029 \\
\hline $\mathrm{RP}$ & 0 & 0 & 0.063 & 0.049 & 0.438 & 0.438 & 0.014 \\
\hline RA & 0 & 0.010 & 0.029 & 0.025 & 0.436 & 0.495 & 0.005 \\
\hline RS & 0 & 0.009 & 0.047 & 0.023 & 0.360 & 0.537 & 0.023 \\
\hline RL & 0 & 0 & 0.034 & 0.053 & 0.403 & 0.471 & 0.039 \\
\hline $\mathrm{RB}$ & 0 & 0.020 & 0.059 & 0.010 & 0.455 & 0.421 & 0.035 \\
\hline $\mathrm{RE}$ & 0 & 0.005 & 0.047 & 0.021 & 0.326 & 0.547 & 0.053 \\
\hline \multicolumn{8}{|c|}{ Bay of Biscay } \\
\hline $\mathrm{CN}$ & 0 & 0.028 & 0.056 & 0 & 0.514 & 0.389 & 0.014 \\
\hline CG & 0 & 0.005 & 0.046 & 0.005 & 0.361 & 0.546 & 0.037 \\
\hline $\mathrm{CL}$ & 0 & 0.006 & 0.075 & 0.013 & 0.381 & 0.481 & 0.044 \\
\hline $\mathrm{CZ}$ & 0 & 0.004 & 0.034 & 0.017 & 0.381 & 0.513 & 0.051 \\
\hline $\mathrm{CP}$ & 0.007 & 0.013 & 0.033 & 0.053 & 0.380 & 0.507 & 0.007 \\
\hline $\mathrm{CH}$ & 0.004 & 0.008 & 0.067 & 0.021 & 0.395 & 0.445 & 0.059 \\
\hline $\mathrm{H} 1$ & 0.004 & 0.025 & 0.182 & 0.025 & 0.352 & 0.403 & 0.008 \\
\hline $\mathrm{H} 2$ & 0 & 0.056 & 0.074 & 0.019 & 0.444 & 0.333 & 0.074 \\
\hline $\mathrm{H} 3$ & 0 & 0.046 & 0.116 & 0.042 & 0.315 & 0.477 & 0.005 \\
\hline H5 & 0.009 & 0.112 & 0.461 & 0.026 & 0.168 & 0.216 & 0.009 \\
\hline H6 & 0.018 & 0.151 & 0.539 & 0.026 & 0.138 & 0.123 & 0.004 \\
\hline $\mathrm{H7}$ & 0 & 0.047 & 0.217 & 0.042 & 0.292 & 0.377 & 0.024 \\
\hline \multicolumn{8}{|c|}{ Northwestern Europe ( $M$. edulis) } \\
\hline ER & 0.007 & 0.257 & 0.693 & 0 & 0.043 & 0 & 0 \\
\hline $\mathrm{EC}$ & 0.025 & 0.108 & 0.825 & 0.025 & 0.017 & 0 & 0 \\
\hline EN & 0.021 & 0.167 & 0.792 & 0 & 0.021 & 0 & 0 \\
\hline
\end{tabular}

multidimensional scaling analysis based upon Euclidean distances of the sample means of the 3 ratios, previously standardized, is shown in Fig. 4. The control samples of $M$. edulis ( $\mathrm{E}_{-}$) were strongly separated from most mussel populations. Some French-Cantabrian samples (H2, H6, $\mathrm{H} 7$ and $\mathrm{H} 8$ ) had intermediate positions between the 2 mussel forms. There were clear-cut morphological differences between $M$. edulis control samples and most of the Iberian mussel populations (M. galloprovincialis), except some FrenchCantabrian mussel populations. On the other hand, there was no evidence of morphological differentiation within $M$. galloprovincialis populations as found in the allozyme data. Thus, the allozyme and morphological data showed a clear-cut difference between Mediterranean $M$. galloprovincialis and northwestern European $M$. edulis. Also, the samples from the Lusitanian, Galician and Hispano-Cantabrian coasts presented morphological and allozyme characters similar to $\mathcal{M}$. galloprovincialis, and some mussel samples from the French-Cantabrian coast exhibited an intermediate position.

An analysis of the genetic structure of the Iberian mussel populations by mean of F-statistics is shown in Table 7. Two aspects are interesting: there were consistent and significant deficiencies of heterozygotes in some cases which are mainly associated with the locus $O d h^{*}$. Second, only samples from the French Atlantic 


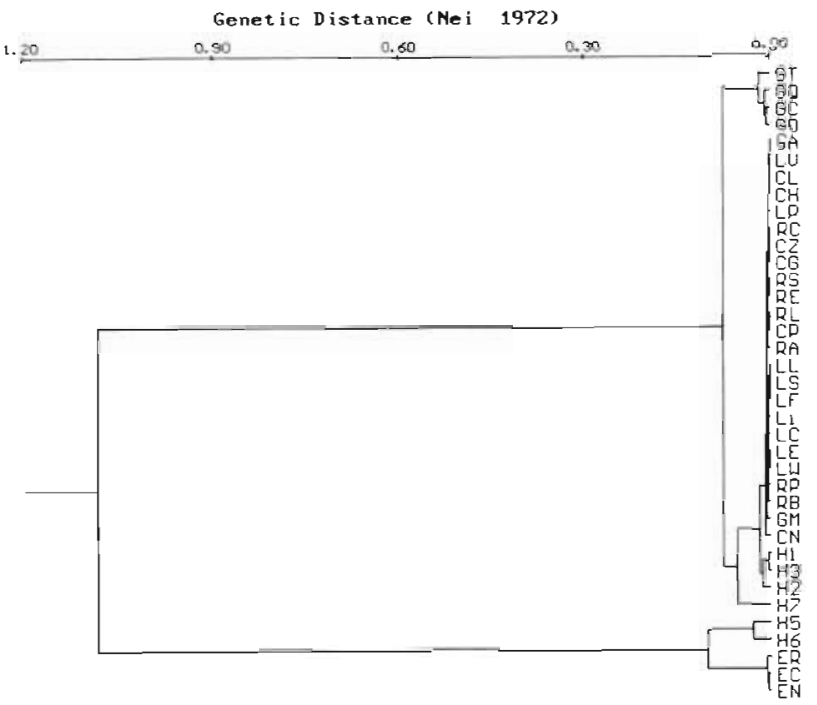

Fig. 2. Mytilus spp. Dendrogram showing genetic relationships among sampled populations (Mediterranean: $\mathrm{G}_{-}$; Lusitanian: L_; Galician Rías: $R_{-}$; Hispano-Cantabrian: $\mathrm{C}_{-}$; French-Cantabrian: $\mathrm{H}_{-}$; control $M$. edulis: $\left.\mathrm{E}_{-}\right)$. Nei's genetic distance from allozyme frequencies at 5 loci were clustered using unweighted pair-group method of analysis (UPGMA). Cophenetic correlation of dendrogram is $\mathrm{r}=0.876$. Population codes as in Table 1

coast ( $\mathrm{H} 1$ to $\mathrm{H6}$ ) presented significant deficiencies of heterozygotes for at least 3 of the 4 best diagnostic loci (Est-D*, Lap-1*, Mpi* and Odh'). This can be explained by a mixture of 2 populations with different allozyme frequencies (Wahlund effect).

A multilocus analysis can also be informative about the genetic composition of the mussel populations. Associations by the chi-square test for different 2-locus genotype combinations were calculated. ' $E$ ' and ' $G$ ' composite alleles were considered. Allele ' $\mathrm{E}$ ' by locus was Ap-1*100, Est-D*100, Lap-1*96 and Lap-1•100, Mpi ${ }^{*} 200$, and $O d h * 115$, and allele 'G' was Ap-1 108 , Est-D•90, Lap-1•104 and Lap-1·108, Mpi・100, and Odh 100 and $\operatorname{Odh} * 129$. A low percentage of the chisquare tests were significant in the Mytilus galloprovincialis $(11 / 57=19.3 \%)$ and $M$. edulis $(2 / 20=$ $10.0 \%)$ control populations, and in the Lusitanian $(6 / 86$ $=7.0 \%)$, Galician $(9 / 70=12.9 \%)$ and Hispano-French $(5 / 59=8.5 \%)$ populations, whereas a large number of association chi-square values $(40 / 50=80.0 \%)$ were highly significant in the French-Cantabrian populations. Moreover, only French-Cantabrian populations (H_) showed a highly significant chi-square association tests ( $p<0.001$ ) for the combinations of the 4 best diagnostic loci. These results imply either a significant excess of double homozygote genotypes for 2 loci (E/E $\mathrm{E} / \mathrm{E}$ or $\mathrm{G} / \mathrm{G} \mathrm{G} / \mathrm{G}$ ) and/or a deficit of double heterozygotes (E/G E/G) and suggest, therefore, a mixture of $M$. edulis and $M$. galloprovincialis populations. Other
Table 5. Mytilus spp. Allele frequencies at the $M p i^{*}$ locus for Iberian Peninsula mussel populations and for typical $M$. edulis

\begin{tabular}{|c|c|c|c|c|}
\hline Population & 25 & 100 & 200 & 300 \\
\hline \multicolumn{5}{|c|}{ Mediterranean coasts (M. galloprovincialis) } \\
\hline GT & 0.012 & 0.927 & 0.061 & 0 \\
\hline $\mathrm{GQ}$ & 0 & 0.973 & 0.027 & 0 \\
\hline $\mathrm{GC}$ & 0 & 0.962 & 0.038 & 0 \\
\hline $\mathrm{GD}$ & 0 & 0.941 & 0.059 & 0 \\
\hline GA & 0.006 & 0.945 & 0.049 & 0 \\
\hline GM & 0.013 & 0.941 & 0.046 & 0 \\
\hline \multicolumn{5}{|c|}{ Lusitanian coasts } \\
\hline LV & 0 & 0.956 & 0.044 & 0 \\
\hline LL & 0.014 & 0.950 & 0.037 & 0 \\
\hline LC & 0.023 & 0.931 & 0.046 & 0 \\
\hline LS & 0.018 & 0.936 & 0.045 & 0 \\
\hline $\mathrm{Li}$ & 0.007 & 0.936 & 0.050 & 0.007 \\
\hline LE & 0.014 & 0.972 & 0.014 & 0 \\
\hline LP & 0 & 0.935 & 0.065 & 0 \\
\hline LF & 0.005 & 0.937 & 0.059 & 0 \\
\hline LW & 0.009 & 0.973 & 0.018 & 0 \\
\hline \multicolumn{5}{|c|}{ Galician Rías } \\
\hline $\mathrm{RC}$ & 0.007 & 0.929 & 0.064 & 0 \\
\hline RP & 0.008 & 0.944 & 0.040 & 0.008 \\
\hline RA & 0.022 & 0.888 & 0.084 & 0.006 \\
\hline $\mathrm{RS}$ & 0.009 & 0.939 & 0.051 & 0 \\
\hline RL & 0 & 0.919 & 0.081 & 0 \\
\hline $\mathrm{RB}$ & 0.010 & 0.933 & 0.053 & 0.005 \\
\hline $\mathrm{RE}$ & 0.005 & 0.940 & 0.055 & 0 \\
\hline \multicolumn{5}{|c|}{ Bay of Biscay } \\
\hline$C N$ & 0 & 0.971 & 0.029 & 0 \\
\hline CG & 0.005 & 0.936 & 0.059 & 0 \\
\hline$C L$ & 0.006 & 0.944 & 0.050 & 0 \\
\hline $\mathrm{CZ}$ & 0 & 0.962 & 0.038 & 0 \\
\hline$C P$ & 0 & 0.933 & 0.067 & 0 \\
\hline $\mathrm{CH}$ & 0.004 & 0.925 & 0.063 & 0.008 \\
\hline H1 & 0 & 0.849 & 0.151 & 0 \\
\hline $\mathrm{H} 2$ & 0 & 0.833 & 0.167 & 0 \\
\hline H3 & 0.009 & 0.809 & 0.182 & 0 \\
\hline $\mathrm{H} 5$ & 0 & 0.439 & 0.553 & 0.009 \\
\hline H6 & 0.004 & 0.314 & 0.672 & 0.009 \\
\hline $\mathrm{H} 7$ & 0.009 & 0.705 & 0.282 & 0.005 \\
\hline \multicolumn{5}{|c|}{ Northwestern Europe ( $M$. edulis) } \\
\hline ER & 0 & 0.059 & 0.941 & 0 \\
\hline $\mathrm{EC}$ & 0.007 & 0.049 & 0.875 & 0.069 \\
\hline EN & 0.010 & 0.020 & 0.970 & 0 \\
\hline
\end{tabular}

samples from the Iberian coast showed varying significant association values which depended of the absence or the low values of ' $E / E$ ' or ' $E / G$ ' double genotypes.

The elaboration of a hybrid index or composite genetic index allowed an analysis of the populations at the individual level (Fig. 5). The hybrid index values of Mytilus edulis control samples (EN, ER) exceeded 0 (1 to 8 ) and the Mediterranean samples of $M$. galloprovincialis ranged from 0 to -8 (Fig. $5 \mathrm{a}$ ). The Lusitanian ( $\mathrm{L}_{-}$), Galician (R_) and Hispano-Cantabrian (C_) samples (except Hondarribia, $\mathrm{CH}$ ) showed exclusively hybrid index values corresponding to the M. gallo- 
Table 6. Mytilus spp. Allele frequencies at the Odh ${ }^{*}$ locus for Iberian Peninsula mussel populations and for typical $M$. edulis

\begin{tabular}{|c|c|c|c|c|c|c|c|c|c|}
\hline Population & 80 & 95 & 100 & 102 & 112 & 115 & 120 & 129 & 140 \\
\hline \multicolumn{10}{|c|}{ Mediterranean coasts (M. galloprovincialis) } \\
\hline GT & 0 & 0 & 0.211 & 0 & 0 & 0.316 & 0 & 0.474 & 0 \\
\hline $\mathrm{GQ}$ & 0.007 & 0 & 0.103 & 0 & 0 & 0.288 & 0 & 0.603 & 0 \\
\hline GC & 0.015 & 0 & 0.146 & 0 & 0 & 0.192 & 0 & 0.646 & 0 \\
\hline GD & 0.007 & 0 & 0.162 & 0 & 0 & 0.140 & 0 & 0.691 & 0 \\
\hline GA & 0 & 0 & 0.677 & 0 & 0 & 0.104 & 0 & 0.213 & 0.006 \\
\hline GM & 0.007 & 0 & 0.651 & 0 & 0 & 0.066 & 0 & 0.270 & 0.007 \\
\hline \multicolumn{10}{|c|}{ Lusitanian coasts } \\
\hline LV & 0.019 & 0 & 0.625 & 0 & 0 & 0.112 & 0 & 0.244 & 0 \\
\hline LL & 0 & 0 & 0.527 & 0.005 & 0.023 & 0.082 & 0 & 0.359 & 0.005 \\
\hline LC & 0.016 & 0 & 0.508 & 0 & 0 & 0.090 & 0 & 0.377 & 0.008 \\
\hline LS & 0.005 & 0 & 0.523 & 0 & 0.014 & 0.130 & 0 & 0.329 & 0 \\
\hline $\mathrm{Li}$ & 0 & 0 & 0.500 & 0 & 0 & 0.100 & 0.014 & 0.379 & 0.007 \\
\hline LE & 0.005 & 0 & 0.542 & 0 & 0 & 0.126 & 0 & 0.308 & 0.019 \\
\hline LP & 0.010 & 0 & 0.638 & 0.005 & 0 & 0.117 & 0 & 0.230 & 0 \\
\hline LF & 0.009 & 0 & 0.523 & 0 & 0 & 0.149 & 0.005 & 0.306 & 0.009 \\
\hline $\mathrm{LW}$ & 0.009 & 0 & 0.564 & 0 & 0.005 & 0.095 & 0.005 & 0.323 & 0 \\
\hline \multicolumn{10}{|c|}{ Galician Rías } \\
\hline $\mathrm{RC}$ & 0.004 & 0.004 & 0.568 & 0.004 & 0.004 & 0.129 & 0 & 0.289 & 0 \\
\hline $\mathrm{RP}$ & 0 & 0 & 0.500 & 0 & 0.005 & 0.149 & 0 & 0.346 & 0 \\
\hline $\mathrm{RA}$ & 0.005 & 0 & 0.518 & 0 & 0 & 0.191 & 0 & 0.264 & 0.023 \\
\hline RS & 0.005 & 0 & 0.528 & 0 & 0 & 0.159 & 0 & 0.308 & 0 \\
\hline RL & 0 & 0 & 0.578 & 0.005 & 0.009 & 0.128 & 0 & 0.280 & 0 \\
\hline $\mathrm{RB}$ & 0.020 & 0 & 0.574 & 0 & 0.004 & 0.098 & 0.008 & 0.283 & 0.012 \\
\hline RE & 0 & 0 & 0.535 & 0 & 0 & 0.131 & 0.005 & 0.328 & 0 \\
\hline \multicolumn{10}{|c|}{ Bay of Biscay } \\
\hline $\mathrm{CN}$ & 0 & 0 & 0.500 & 0 & 0.014 & 0.114 & 0 & 0.329 & 0.043 \\
\hline CG & 0.014 & 0 & 0.586 & 0 & 0 & 0.109 & 0 & 0.273 & 0.018 \\
\hline CL & 0.003 & 0 & 0.615 & 0 & 0.003 & 0.127 & 0 & 0.252 & 0 \\
\hline $\mathrm{CZ}$ & 0 & 0 & 0.593 & 0 & 0.004 & 0.102 & 0 & 0.301 & 0 \\
\hline $\mathrm{CP}$ & 0 & 0 & 0.588 & 0 & 0 & 0.095 & 0 & 0.311 & 0.007 \\
\hline $\mathrm{CH}$ & 0 & 0 & 0.621 & 0 & 0.004 & 0.162 & 0 & 0.208 & 0.004 \\
\hline $\mathrm{H} 1$ & 0.004 & 0 & 0.471 & 0 & 0.008 & 0.225 & 0.004 & 0.287 & 0 \\
\hline $\mathrm{H} 2$ & 0.017 & 0 & 0.567 & 0 & 0 & 0.150 & 0 & 0.267 & 0 \\
\hline H3 & 0 & 0 & 0.505 & 0 & 0.005 & 0.220 & 0 & 0.266 & 0.005 \\
\hline H5 & 0.017 & 0 & 0.291 & 0 & 0 & 0.543 & 0 & 0.141 & 0.009 \\
\hline H6 & 0.007 & 0 & 0.223 & 0.004 & 0.004 & 0.677 & 0 & 0.080 & 0.004 \\
\hline $\mathrm{H} 7$ & 0.005 & 0 & 0.468 & 0 & 0.005 & 0.286 & 0 & 0.232 & 0.005 \\
\hline \multicolumn{10}{|c|}{ Northwestern Europe ( $M$. edulis) } \\
\hline ER & 0 & 0 & 0.088 & 0 & 0 & 0.868 & 0 & 0.044 & 0 \\
\hline EC & 0 & 0 & 0.040 & 0 & 0 & 0.960 & 0 & 0 & 0 \\
\hline EN & 0 & 0 & 0.021 & 0 & 0 & 0.947 & 0 & 0.021 & 0.011 \\
\hline
\end{tabular}

provincialis range. The samples from Hondarribia $(\mathrm{CH})$ and the French-Cantabrian coast $\left(\mathrm{H}_{-}\right.$, Barre de L'Adour and Capbreton) exhibited values in the range of $M$. edulis and $M$. galloprovincialis as well as individuals with 0 value in a varying proportion (Fig. $5 b, c$, d). Most individuals with 0 value from Hondarribia and French-Cantabrian coast, where there are $M$. edulis and $M$. galloprovincialis individuals, were ' $E / G$ ' tetraheterozygotes and therefore presumably hybrids of both mussel forms. In contrast, individuals with a hybrid index of 0 from other Atlantic populations and from Mediterranean samples were never ' $\mathrm{E} / \mathrm{G}$ ' tetraheterozygotes

\section{DISCUSSION}

Enzyme polymorphisms and morphological variation have revealed the ubiquitous presence of Mytilus galloprovincialis on the Iberian coast. The allozyme frequencies for the best diagnostic loci (Est- $D^{*}, \operatorname{Lap} \mathbf{1}^{*}$, $M^{*} i^{*}$ and $O d h^{*}$; Tables 3 to 6) and the UPGMA dendrogram and nonmetric multidimensional scaling from genetic and morphological distances (Figs. 2, 3 \& 4) showed the clustering of most of Iberian mussel populations with pure $M$. galloprovincialis populations from the Mediterranean coast. The concordance between both morphological variation and enzyme polymor- 

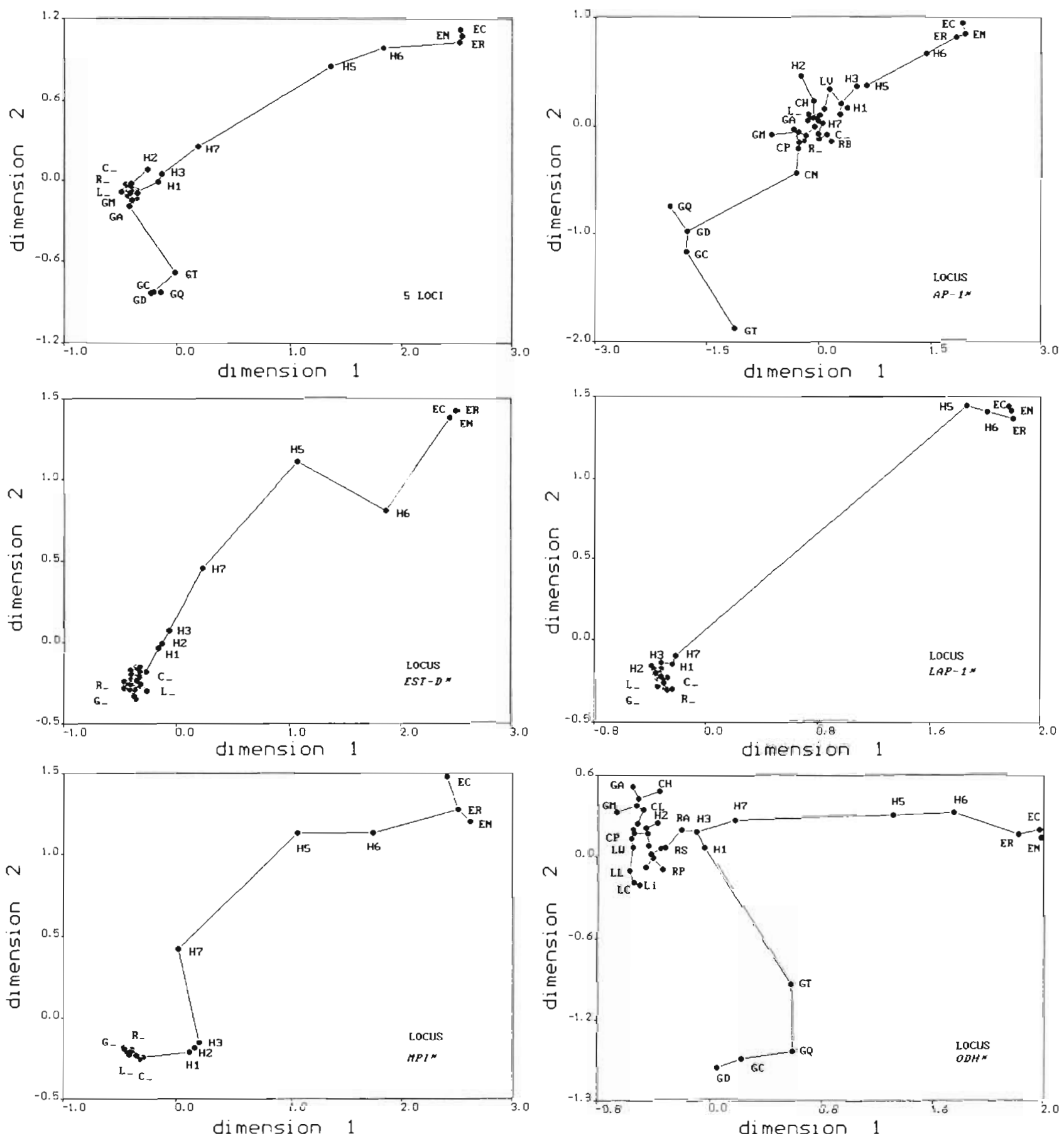

Fig. 3. Mytilus spp. Ordination of sampled populations in 2 dimensions by non-metric multidimensional scalıng (MDS) of Nei's genetic distances calculated among all pairs of populations for the 5 loci, as well as for each locus separately (Ap-1*, Est- $D^{*}$, Lap-1*, Mpi and Odh*). Stress of each MDS plot is 0.020, 0.076, 0.006, 0.007, 0.004, and 0.009 respectively. Minimum spanning tree is superimposed. Population codes as in Table 1

phisms in the present work is virtually complete and indicates that $M$. galloprovincialis commonly occurs along the coasts of the Iberian Peninsula: the Mediterranean, Lusitanian, Galician and Bay of Biscay coasts. These results from the Atlantic Iberian coast permit a fuller understanding of the data of Sanjuan et al. (1986, 1990), which show the presence of $M$. galloprovincialis in the NW Iberian area, and some partial data in other scattered locations (Verduin 1979, Bulnheim \& Gosling 1988, M.cDonald et al. 1991). The present investigation 


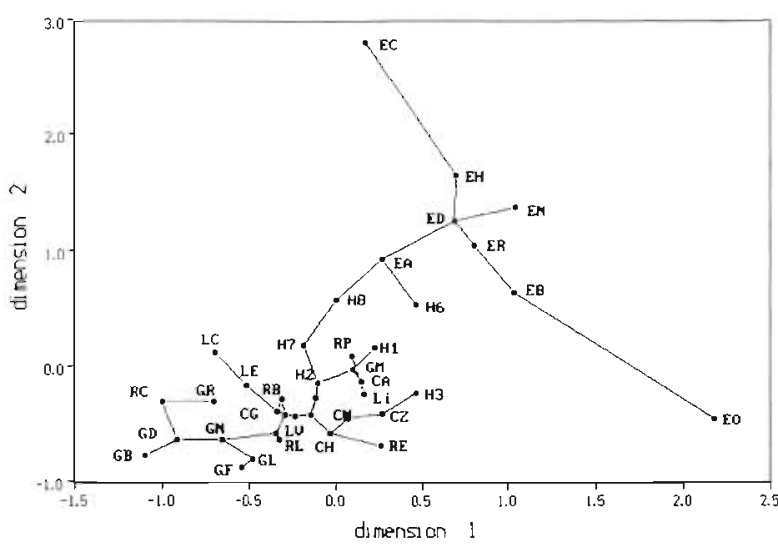

Fig. 4. Mytilus spp. Ordination of sampled populations in 2 dimensions by non-metric multidimensional scaling (MDS) of Euclidean distances calculated among all pairs of populations for the 3 conchological ratios. Stress is 0.099 , which is a good goodness of fit. Minimum spanning tree is superimposed. Population codes as in Table 1

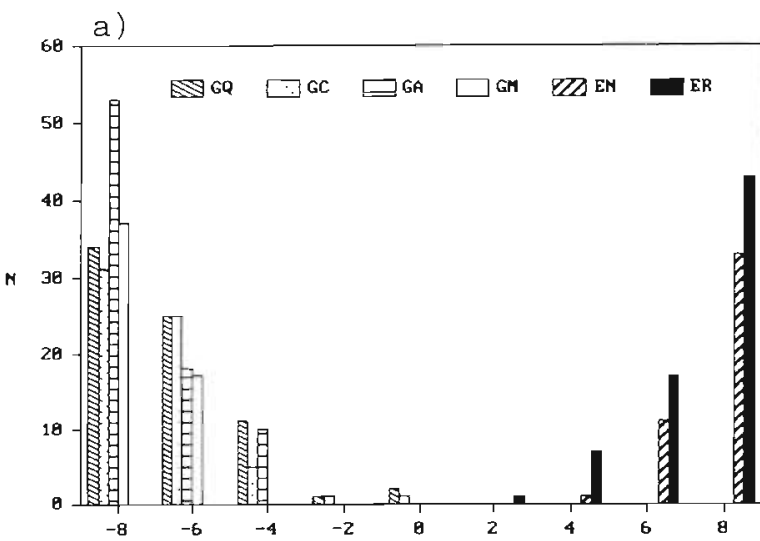

b)

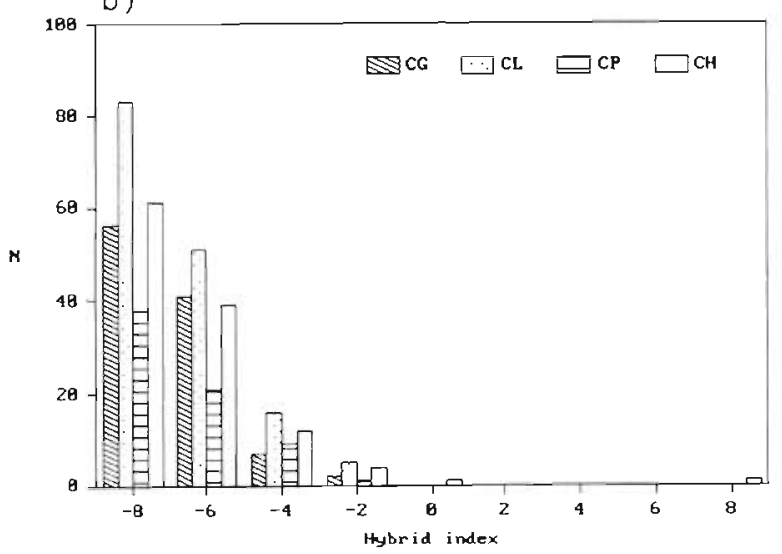

shows a continuous distribution of the M. galloprovincialis form from the Mediterranean Sea to the English Channel and British Isles. This continuous distribution of $M$. galloprovincialis seems to eliminate the possibility that British $M$. galloprovincialis populations evolved from an $M$. edulis-like ancestral population (Skibinski et al. 1978, 1983) and it suggests instead a migration and northward extension from the Mediterranean populations as suggested by Barsotti \& Meluzzi (1968).

On the other hand, there was no evidence of Mytilus edulis, or hybrids, in mussel populations from the Mediterranean, Lusitanian, Galician and HispanoCantabrian (except in Hondarribia, $\mathrm{CH}$ ) coasts, and the presence of $M$. edulis was only detected in samples near the Hispano-French frontier. In general, the UPGMA dendrogram and the nonmetric multidimensional scaling from genetic distances (Figs. $2 \& 3$ respectively) show that mussel populations of that

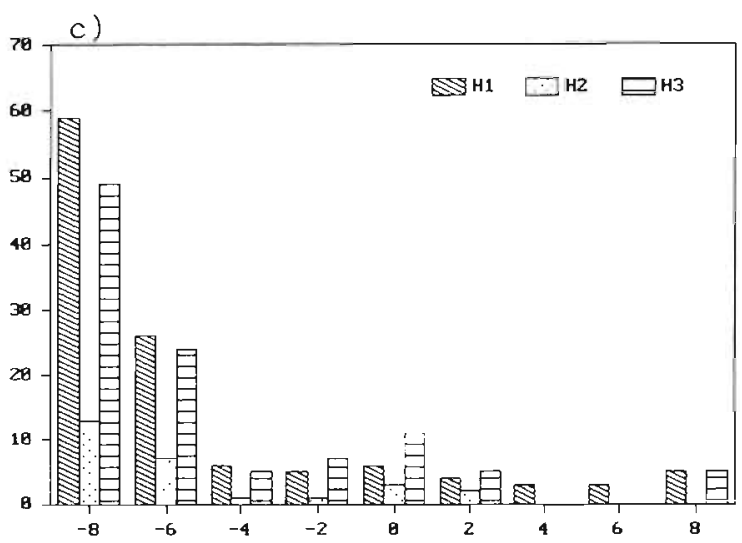

d)

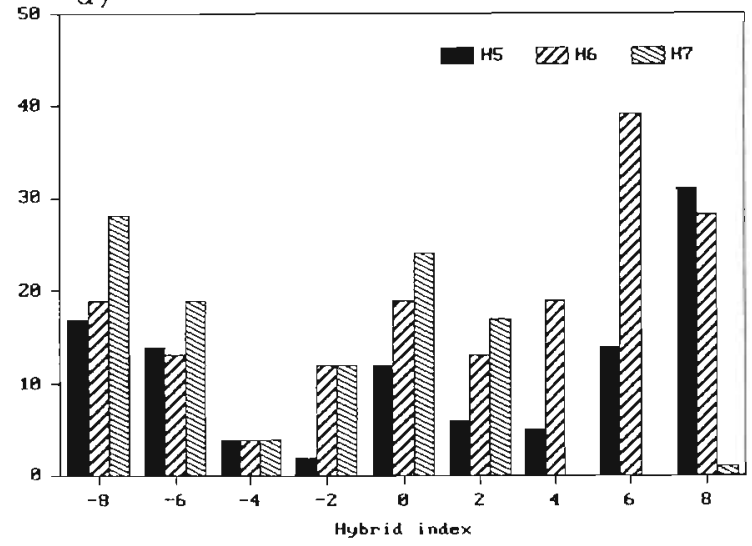

Fig. 5. Mytilus spp. Histograms showing distributions of the composite genetic or hybrid index in: (a) Mediterranean M. galloprovincialis (G_) and Atlantic $M$. edulis ( $\mathrm{E}_{-}$) control populations; (b) Hispano-Cantabrian populations (C_); (c) 3 samples from Barre de L'Adour (H1, H2 and H3); and (d) 3 samples from Capbreton (H5, H6 and H7). To obtain the hybrid index, +1 was assigned to each typical allele of $M$. edulis, -1 to each typical allele of $M$. galloprovincialis, and 0 to others. The score for each individual is the sum of values across the 4 diagnostic loci (Est- $D^{*}$, Lap- $1^{\circ}$, Mpi $i^{*}$ and Odh'). Each class is constituted by individuals with 2 consecutive hybrid index values (e.g. class -8 groups individuals with -8 and -7 scores), except class 0 which only contain individuals with 0 score for hybrid index. N: number of individuals scored 
Table 7. Mytilus spp. Estimates of the F-statistics for the mussel populations from the Iberian Peninsula. $F_{S T}{ }^{\prime}$ and $F_{r r^{\prime}}$ are Wright's fixation indices estimated after Nei \& Chesser (1983), and $F$ in each sample is estimated after Robertson \& Hill (1984) with standard error. $M$. edulis $\left(E_{-}\right)$and hybrid zone $(\mathrm{H} 1$ to $\mathrm{H} 7)$ populations were not considered in calculating $F_{I S}, F_{S T}$, $F_{\Gamma T}^{\prime}$ and homogeneity $\chi^{2}$ tests, $p<0.05_{i} \cdots p<0.01_{i} \cdots p<0.001$

\begin{tabular}{|c|c|c|c|c|c|}
\hline Population & $\begin{array}{l}A p-1^{\circ} \\
F \pm \mathrm{SE}\end{array}$ & $\begin{array}{l}E s t-D^{\cdot} \\
F \pm S E\end{array}$ & $\begin{array}{l}L a p-1 \cdot \\
F \pm S E\end{array}$ & $\begin{array}{c}M p i^{\bullet} \\
F \pm \mathrm{SE}\end{array}$ & $\begin{array}{l}O d h^{*} \\
F \pm \mathrm{SE}\end{array}$ \\
\hline \multicolumn{6}{|c|}{ Mediterranean coasts (M. galloprovincialis) } \\
\hline GT & $0.288 \pm 0.090^{\cdots}$ & $0.165 \pm 0.110$ & $-0.098 \pm 0.090$ & $-0.055 \pm 0.156$ & $0.528 \pm 0.115 \cdots$ \\
\hline $\mathrm{GQ}$ & $-0.003 \pm 0.068$ & $-0.031 \pm 0.116$ & $-0.090 \pm 0.116$ & $-0.021 \pm 0.116$ & $0.271 \pm 0.083^{\cdots}$ \\
\hline $\mathrm{GC}$ & $-0.019 \pm 0.072$ & $-0.030 \pm 0.072$ & $0.055 \pm 0.063$ & $-0.033 \pm 0.124$ & $0.075 \pm 0.088$ \\
\hline GD & $0.044 \pm 0.070$ & $-0.016 \pm 0.121$ & $-0.023 \pm 0.061$ & $0.212 \pm 0.121$ & $-0.082 \pm 0.086$ \\
\hline $\mathrm{GA}$ & $0.012 \pm 0.064$ & $-0.035 \pm 0.110$ & $0.092 \pm 0.064$ & $-0.046 \pm 0.110$ & $0.160 \pm 0.078^{*}$ \\
\hline GM & $0.106 \pm 0.066$ & $-0.061 \pm 0.114$ & $-0.020 \pm 0.134$ & $-0.043 \pm 0.115$ & $-0.007 \pm 0.081$ \\
\hline \multicolumn{6}{|c|}{ Lusitanian coasts } \\
\hline LV & $-0.124 \pm 0.065$ & $-0.028 \pm 0.079$ & $0.141 \pm 0.065^{\circ}$ & $-0.040 \pm 0.112$ & $0.091 \pm 0.079$ \\
\hline LL & $0.012 \pm 0.055$ & $0.025 \pm 0.056$ & $0.101 \pm 0.056$ & $0.226 \pm 0.096^{\circ}$ & $0.252 \pm 0.067 \cdots$ \\
\hline LC & $0.066 \pm 0.071$ & $-0.094 \pm 0.125$ & $0.062 \pm 0.088$ & $-0.044 \pm 0.124$ & $0.121 \pm 0.091$ \\
\hline LS & $-0.007 \pm 0.055$ & $0.176 \pm 0.067 \cdots$ & $-0.048 \pm 0.067$ & $-0.045 \pm 0.095$ & $0.120 \pm 0.068$ \\
\hline $\mathrm{Li}$ & $0.013 \pm 0.069$ & $-0.065 \pm 0.085$ & $0.112 \pm 0.120$ & $-0.048 \pm 0.120$ & $0.026 \pm 0.085$ \\
\hline LE & $-0.079 \pm 0.068$ & $-0.037 \pm 0.068$ & $0.202 \pm 0.100^{\circ}$ & $-0.024 \pm 0.097$ & $0.151 \pm 0.068^{\circ}$ \\
\hline $\mathrm{LP}$ & $0.118 \pm 0.058^{\circ}$ & $-0.044 \pm 0.071$ & $0.132 \pm 0.071$ & $0.266 \pm 0.100^{\circ}$ & $0.224 \pm 0.074^{\cdots}$ \\
\hline LF & $0.050 \pm 0.055$ & $0.070 \pm 0.067$ & $0.172 \pm 0.055^{*}$ & $0.115 \pm 0.095$ & $0.205 \pm 0.067^{\cdots}$ \\
\hline LW & $-0.036 \pm 0.055$ & $-0.047 \pm 0.103$ & $0.105 \pm 0.055$ & $-0.024 \pm 0.095$ & $0.052 \pm 0.067$ \\
\hline \multicolumn{6}{|c|}{ Galician Rías } \\
\hline $\mathrm{RC}$ & $0.040 \pm 0.049$ & $0.049 \pm 0.060$ & $-0.023 \pm 0.060$ & $-0.066 \pm 0.085$ & $0.110 \pm 0.060$ \\
\hline $\mathrm{RP}$ & $0.105 \pm 0.063$ & $-0.023 \pm 0.103$ & $-0.089 \pm 0.068$ & $-0.035 \pm 0.126$ & $0.241 \pm 0.073^{\cdots}$ \\
\hline RA & $-0.018 \pm 0.056$ & $0.091 \pm 0.098$ & $0.058 \pm 0.099$ & $-0.080 \pm 0.106$ & $0.154 \pm 0.067^{\circ}$ \\
\hline RS & $-0.013 \pm 0.057$ & $-0.046 \pm 0.097$ & $0.041 \pm 0.068$ & $0.142 \pm 0.097$ & $0.254 \pm 0.068 \cdots$ \\
\hline $\mathrm{RL}$ & $0.048 \pm 0.056$ & $0.084 \pm 0.068$ & $0.202 \pm 0.049 \cdots$ & $0.174 \pm 0.098$ & $0.186 \pm 0.068^{\cdots}$ \\
\hline RB & $0.161 \pm 0.052$ & $-0.042 \pm 0.099$ & $-0.010 \pm 0.057$ & $-0.053 \pm 0.098$ & $0.106 \pm 0.064$ \\
\hline $\mathrm{RE}$ & $-0.010 \pm 0.058$ & $-0.040 \pm 0.100$ & $-0.078 \pm 0.059$ & $0.139 \pm 0.100$ & $-0.040 \pm 0.071$ \\
\hline \multicolumn{6}{|c|}{ Bay of Biscay } \\
\hline $\mathrm{CN}$ & $0.035 \pm 0.096$ & $0.519 \pm 0.167^{\circ}$ & $0.465 \pm 0.118 \cdots$ & $-0.015 \pm 0.169$ & $-0.170 \pm 0.098$ \\
\hline $\mathrm{CG}$ & $0.101 \pm 0.067$ & $-0.044 \pm 0.067$ & $-0.065 \pm 0.056$ & $-0.059 \pm 0.095$ & $0.130 \pm 0.067$ \\
\hline $\mathrm{CL}$ & $-0.048 \pm 0.046$ & $0.029 \pm 0.056$ & $-0.017 \pm 0.046$ & $0.082 \pm 0.079$ & $0.224 \pm 0.056 \cdots$ \\
\hline $\mathrm{CZ}$ & $-0.002 \pm 0.053$ & $-0.045 \pm 0.065$ & $0.066 \pm 0.053$ & $0.197 \pm 0.091^{\circ}$ & $0.136 \pm 0.065^{\circ}$ \\
\hline $\mathrm{CP}$ & $-0.022 \pm 0.066$ & $0.065 \pm 0.081$ & $0.073 \pm 0.067$ & $-0.065 \pm 0.116$ & $0.110 \pm 0.082$ \\
\hline $\mathrm{CH}$ & $-0.037 \pm 0.053$ & $-0.044 \pm 0.091$ & $0.043 \pm 0.053$ & $0.078 \pm 0.091$ & $0.071 \pm 0.065$ \\
\hline $\mathrm{H} 1$ & $0.092 \pm 0.065$ & $0.225 \pm 0.065 \cdots$ & $0.251 \pm 0.065 \cdots$ & $0.417 \pm 0.092 \cdots$ & $0.229 \pm 0.065 \cdots$ \\
\hline $\mathrm{H} 3$ & $0.035 \pm 0.056$ & $0.197 \pm 0.095^{\circ}$ & $0.103 \pm 0.048^{\circ}$ & $0.084 \pm 0.095$ & $0.163 \pm 0.068^{\circ}$ \\
\hline $\mathrm{H} 5$ & $0.100 \pm 0.065$ & $0.443 \pm 0.090 \cdots$ & $0.244 \pm 0.054 \cdots$ & $0.585 \pm 0.093 \cdots$ & $0.258 \pm 0.065 \cdots$ \\
\hline $\mathrm{H} 6$ & $0.021 \pm 0.047$ & $0.308 \pm 0.066 \cdots$ & $0.116 \pm 0.038^{*}$ & $0.419 \pm 0.066^{\cdots}$ & $0.175 \pm 0.047^{\cdots}$ \\
\hline $\mathrm{H7}$ & $0.098 \pm 0.055$ & $-0.098 \pm 0.067$ & $-0.023 \pm 0.049$ & $-0.254 \pm 0.095^{\cdots}$ & $0.111 \pm 0.067$ \\
\hline \multicolumn{6}{|c|}{ Northwestern Europe ( $M$. edulis) } \\
\hline ER & $0.181 \pm 0.122$ & $-0.032 \pm 0.120$ & $0.140 \pm 0.085$ & $0.212 \pm 0.121$ & $0.116 \pm 0.086$ \\
\hline $\mathrm{EC}$ & $0.062 \pm 0.083$ & $-0.026 \pm 0.121$ & $0.038 \pm 0.129$ & $0.091 \pm 0.083$ & $0.386 \pm 0.127^{\cdots}$ \\
\hline EN & $0.178 \pm 0.141$ & $-0.049 \pm 0.141$ & $0.579 \pm 0.144 \cdots$ & $-\quad-$ & $0.337 \pm 0.084 \cdots$ \\
\hline$F_{I S}$ & 0.028 & -0.001 & 0.065 & 0.022 & 0.127 \\
\hline$F_{I T}^{\prime}$ & 0.048 & 0.009 & 0.073 & 0.028 & 0.187 \\
\hline$F_{S T}$ & 0.021 & 0.010 & 0.009 & 0.006 & 0.069 \\
\hline$x^{2}$ & $230.8^{\cdots}$ & $40.7^{\circ}$ & $75.3^{\circ}$ & 30.1 & $419.3 \cdots$ \\
\hline
\end{tabular}

area $\left(\mathrm{CH}\right.$, Hondarribia, and $\mathrm{H}_{-}$, Barre de L'Adour and Capbreton) exhibited intermediate positions between pure populations of $M$. edulis and Atlantic $M$. galloprovincialis. Intermediate allozyme frequencies for the diagnostic loci between those characteristic of pure $M$. edulis and $M$. galloprovincialis (Tables 2 to 6), consistent and significant deficits of heterozygotes for 3 of the 4 best diagnostic loci $\left(E s t-D^{*}, L a p-1^{\circ}, M p i^{*}\right.$ and Odh': Table 7 ), and highly significant associations between the 4 best diagnostic loci are all evidence for the mixture of 2 genetic pools, i.e. the coexistence of $M$. edulis and $M$. galloprovincialis in varying proportions. Moreover the hybrid index showed typical M. edulis and $M$. galloprovincialis individuals as well 
as hybrids ( $E / G$ tetraheterozygotes) of $M$ edulis and M. galloprovincialis in the samples from this same area $\left(\mathrm{CH}\right.$, Hondarribia, and $\mathrm{H}_{-}$, Barre l'Adour and Capbreton; Fig. 5). The coexistence of these Mytilus forms on the Atlantic coast of France is in accordance with other morphological (Lubet 1959, 1973, Seed 1972, Verduin 1979, Lubet et al. 1984) and allozyme data (Coustau et al. 1991, McDonald et al. 1991). Consequently, the meridional limit of the distribution of European $M$. edulis, and the concomitant limit of the hybrid zone between $M$. edulis and $M$. galloprovincialis, can be accurately established between the mouths of the Bidasoa and Nervion rivers in Spain. This finding contradicts the previously held and widespread opinion that European $M$. edulis ranges from the Arctic to North Africa (see Seed 1976, 1978, 1992, Gosling 1984, Suchanek 1985, Gardner 1992). Only McDonald et al. (1991) cited allozyme evidence of hybrids (and M. galloprovincialis) in a southern location (Parede), but their study did not provide strong evidence for the extension of the hybrid zone because no evidence of $M$. edulis was found and these must be present in order to maintain a hybrid zone (an accidental or artificial introduction could explain those data).

The frequency of characteristic allozymes of Mytilus edulis and $M$. galloprovincialis forms in the hybrid zone of the Bay of Biscay (Tables 2 to 6 ) and the pattern of the hybrid index (Fig. 5) seem to be dependent on the location ( $\mathrm{CH}$, Hondarribia, or $\mathrm{H}_{-}$, Barre de L'Adour or Capbreton) as well as on environmental factors such as the wave exposure (channel or groynes) or the attachment height of the mussel zone, as is found in other areas of the hybrid zone (Gosling \& Wilkins 1981, Skibinski 1983, Skibinski et al. 1983, Gardner \& Skibinski 1988, 1991, Willis \& Skibinski 1992; see Gosling $1992 \mathrm{~b}$ for a review). A more detailed analysis of this hybrid area will be published elsewhere.

Another feature worthy of mention is that Mytilus galloprovincialis from the southern Mediterranean exhibits an abrupt discontinuity in allozyme frequencies of the loci $A p-1^{*}$ and $O d h^{\circ}$. The mean frequencies $( \pm \mathrm{SE})$ for some common allozymes at the $O d h^{\circ}$ and $A p-1^{*}$ loci (in the 4 samples from the northeastern Iberian coasts and in the 24 samples from southern and Atlantic Iberian coasts respectively) were as follows: Odh *100, $0.566 \pm 0.011$ and $0.156 \pm 0.022 ;$ Odh *129, $0.296 \pm 0.010$ and $0.604 \pm 0.047 ; \mathrm{Ap}-1 \cdot 100,0.423 \pm$ 0.008 and $0.176 \pm 0.008$. The frequency of these allozymes followed a stepped cline pattern with mean variations of about 40,30 and $25 \%$ for Odh 100 , Odh*129, and Ap-1*100, respectively (Tables 2 \& 6). A highly significant genetic differentiation among $M$. galloprovincialis was detected at the $A p-1^{*}$ and $O d h^{*}$ loci $\left(F_{S T}\right.$ was 0.021 and 0.069 respectively; Table 7$)$. The 4 samples from the northeastern Iberian coast (GT,
GQ, GC, GD) constituted a small cluster clearly separated from most $M$. galloprovincialis as shown by the UPGMA dendrogram and the non-metric multidimensional plots (Figs. $2 \& 3$ ). This strong genetic differentation within $M$. galloprovincialis over a small geographic distance calls into question the fact that $M$. galloprovincialis can be an homogeneous genetic pool as has been supposed in recent reports (Koehn 1991, McDonald et al. 1991; but see Gosling 1992b). Moreover, it contributes to the explanation of the 'intriguing pattern of variation' of $A p^{*}$ locus (and others) in mussel populations from the European coast (Varvio et al. 1988) and can also help to explain the 'large differences in mtDNA genotype' between British and Mediterranean populations (Ward 1989, Karakousis \& Skibinski 1992).

The area of the Western Mediterrenean Sea where Mytilus galloprovincialis exhibits abrupt discontinuity in allozyme frequencies appears to be associated with an oceanographic current front. The Almeria-Oran front, a strong large-scale ocean front between Almería (SE Spain) and Oran (Algeria) (Tintore et al. 1988, La Violette 1989), could be an important barrier to the migration of the mussel larvae between the 2 sides of this front. These 2 adjacent areas have different biotic and abiotic conditions: the Mediterranean Sea has characteristically warm-temperate floral and faunal assemblages (Rodriguez 1982, Ben-Tuvia 1985, Ketchum 1985, Pérès 1985, Ros et al. 1985, Sara 1985), and the Alboran Sea represents a transition zone between the Atlantic water mass and the inner Mediterranean Sea (Pérès 1967, Estrada et al. 1985, Conde 1990). In addition, no mussels were seen along the intertidal coastal level between Almería and northern Altea (Alicante) during 3 scientific trips to collect mussel samples (Sanjuan 1992), perhaps because the physical (substrate, oceanographic factors) or biological (phytoplankton concentration) conditions of the area are unsuitable for growing mussels. This lack of the appropiate conditions could cause an interruption or reduction of abundance of the mussels. These facts suggest and make plausible a discrete subpopulation model, i.e. European M. galloprovincialis seems subdivided at least into 2 relatively separate genetic pools. An ancient isolation of Mediterranean from Atlantic M. galloprovincialis populations with genetic divergence, followed by secondary contact with a restricted gene flow could explain this stepped cline pattern of allozyme variation (Endler 1977). A similar mechanism has been suggested to account genetic differentiation of Limulus polyphemus (Selander et al. 1970, Saunders et al. 1986) and other estuarine species in southeastern North America (see Avise 1992), and in Platichtys flessus (Galleguillos \& Ward 1982) and Ostrea edulis (Saavedra et al. 1993) between Mediterranean and 
Atlantic populations. The large climatic-eustatic oscillations of the Mediterranean Basin during geological periods, which have been associated with changes in the Mediterranean organism composition (Rodriguez 1982, Maldonado 1985, Pérès 1985, Raffi et al. 1985, Sara 1985, Vermeij 1989), can support this historical hypothesis.

Acknowledgements. We are grateful to $\mathrm{H}$. Quesada and A. Urbieta for technical assistance and to T. Farina, A. Santas and $M . C$. Taboada for assistance in the collection of the mussel samples. This research was supported by grant PAC84-0035 from the Comision Asesora de Investigación Científica y Técnica (CAICYT) of the Ministerio de Educación y Ciencia (Spain) to G.A.

\section{LITERATURE CITED}

Ahmad, M., Beardmore, J. A. (1976). Genetic evidence that the 'Padstow Mussel' is Mytilus galloprovincialis. Mar. Biol. 35: 139-147

Ahmad, M., Skibinski, D. O. F., Beardmore, J. A. (1977). An estimate of the amount of genetic variation in the common mussel Mytilus edulis. Biochem. Genet. 15: 833-846

Avise, J. C. (1992). Molecular population structure and the biogeographic history of a regional fauna: a case history with lessons for conservation biology. Oikos 63: 62-76

Barsotti, G., Meluzzi, C. (1968). Osservazioni su Mytilus edulis L. e M. galloprovincialis Lmk. Conchiglie (Un. malac. ital.) 4: $50-58$

Beaumont, A. R., Seed, R., Garcia-Martinez, P. (1989). Electrophoretic and morphometric criteria for the identification of the mussels Mytilus edulis and $M$. galloprovincialis. In: Ryland, J. S., Tyler, P. A. (eds.) Reproduction, genetics and distributions of marine organisms. Proc. 23rd Eur mar. Biol. Symp. Olsen and Olsen, Fredensborg, p. $251-258$

Ben-Tuvia, A. (1985). The Mediterranean Sea. B. Biological aspects. In: Ketchum, B. H. (ed.) Ecosystems of the world, Vol. 26, Estuaries and enclosed seas. Elsevier Sci. Publ., Amsterdam, p. 239-251

Bulnheim, H.-P., Gosling, E. (1988). Population genetic structure of mussels from the Baltic Sea. Helgoländer. Meeresunters. 42: 113-129

Conde, F. (1990). Ficoecografía del mar de Alborán en el contexto del Mediterráneo Occidental. An. Jardín Bot. Madrid 46: 21-26

Coustau, C., Renaus, F., Delay, B. (1991). Genetic characterization of the hybridization between Mytilus edulis and M. galloprovincialis on the Atlantic coast of France. Mar. Biol. 111:87-93

Dunn, G., Everitt, D. S. (1982). An introduction to mathematical taxonomy. Cambridge Univ. Press, Cambridge

Edwards, C. A., Skibinski, D. O. F. (1987). Genetic variation mitochondrial DNA in mussel (Mytilus edulis and M. galloprovincialis) populations from South West England and South Wales. Mar. Biol. 94: 547-556

Endler, F. A. (1977). Geographic variation, speciation and clines. Princeton Univ. Press, Princeton

Estrada, M., Vives, F., Alcaraz, M. (1985). Life and the productivity of the open sea. In: Margalef, R. (ed.) The Western Mediterranean. Key environment ser. Pergamon Press, Oxford, p. 148-197
Galleguillos, R. A., Ward, R. D. (1982). Genetic and morphological divergence between population of the flatfish Platichtys flesus (L.) (Pleuronectidae). Biol. J. Linn. Soc. 17: $395-408$

Gardner, J. P. (1992). Mytilus galloprovincialis (Lmk.) (Bivalvia, Mollusca): the taxonomic status of the Mediterranean mussel. Ophelia 35: 219-243

Gardner, J. P., Skibinski, D. O. F. (1988). Historical and sizedependent genetic variation in hybrid mussel populations. Heredity 61: 93-105

Gardner, J. P., Skibinski, D. O. F. (1991). Mitochondrial DNA and allozyme co-variation in a hybrid mussel population. J. exp. mar. Biol. Ecol. 149: 45-54

Gosling, E. M. (1984). The systematic status of Mytilus galloprovincialis in Western Europe, a review. Malacologia 25: $551-568$

Gosling, E. M. (1992a). Systematics and geographic distribution of Mytilus. In: Gosling, E. (ed.) The mussel Mytilus: ecology, physiology, genetics and culture. Elsevier, Amsterdam, p. 1-20

Gosling, E. M. (1992b). Genetics of Mytilus, In: Gosling, E. (ed.) The mussel Mytilus: ecology, physiology, genetics and culture. Elsevier, Amsterdam, p. 309-382

Gosling, E. M., Wilkins, N. P. (1981). Ecological genetics of the mussels Mytilus edulis and $M$. galloprovincialis on Irish coasts. Mar. Ecol. Prog. Ser. 4: 221-227

Grant, W. S., Cherry, M. I. (1985). Mytilus galloprovincialis Lmk in Southern Africa. J. exp. mar. Biol. Ecol. 90: 179-191

Harris, H., Hopkinson, D. A. (1976). Handbook of enzyme electrophoresis in human genetics. North-Holland, Amsterdam

Hoeh, W R., Blakley K. H., Brown, W. M. (1991). Heteroplasmy suggests limited biparental inheritance of Mytilus mitochondrial DNA. Science 251: 1488-1490

James, F. C., McCulloch, C. E. (1990). Multivariate analysis in ecology and systematics: panacea or Pandora's box? A. Rev. Ecol. Syst. 21: 129-166

Johannesson, K., Kautsky, N., Tedengren, M. (1990). Genotypic and phenotypic differences between Baltic and North Sea populations of Mytilus edulis evaluated through reciprocal transplantations. II. Genetic variation. Mar. Ecol. Prog. Ser. 59: 211-219

Karakousis, Y., Skibinski, D. O. F. (1992). An analysis of allozyme, mitochondrial DNA and morphological variation in mussel (Mytilus galloprovincialis) populations from Greece. Experientia 48: 878-881

Ketchum, B. (1985). Enclosed seas-introduction. In Ketchum, B. H. (ed.) Ecosystems of the world, Vol. 26, Estuaries and enclosed seas. Elsevier Sci. Publ., Amsterdam, p. 209-218

Koehn, R. K. (1991). The genetics and taxonomy of species in the genus Mytilus. Aquaculture 94: 125-146

La Violette, P. E. (1989). WMCE Western Mediterranean Circulation Experiment: a preliminary review of results EOS Trans. Am. geophys. Un. 70: 756-758

Lewis, J. R., Seed, R. (1969). Morphological variations in Mytilus from South-West England in relation to the occurrence of $M$. galloprovincialis Lamarck. Cah. Biol. mar. 10: $231-253$

Lubet, P. (1959). Recherches sur le cycle sexuel et l'émission des gamètes chez les Mytilides et les Pectinides. Rev. Trav. Inst. (scient. techn.) Pêch. mar. 23: 387-548

Lubet, P. (1973). Exposé synoptique des données biologiques sur la moule Mytilus galloprovincialis (Lamarck 1819). Synopsis FAO sur les pèches No 88 (SAST-Moule, 3, 16 (10). 028, 08, pag. var.). FAO, Rome 
Lubet, P. (1976). L'espèce chez les lamellibranches marins In: Bocquet, C., Genermont, J., Lamotte, M. (eds.) Les problèmes de l'espèce dans le regne animal. Soc. Zool. France, Paris, p. 341-374

Lubet, P., Prunus, G., Masson, M., Bucaille, D. (1984). Recherches experimentales sur l'hybridation de Mytilus edulis L. et M.galloprovincialis Lmk. (Mollusques Lamellibranches). Bull. Soc. Zool. France 109: 87-98

Maldonado, A. (1985). Evolution of the Mediterranean basins and a detailed reconstruction of the Cenozoic paleoceanography. In: Margalef, R. (ed.) Western Mediterranean. Key environment ser. Pergamon Press, Oxford, p. 17-59

McDonald, J. H., Koehn, R. K., Balakirev, E. S., Manchenko, G. P., Pudovkin, A. 1., Sergiyevskii, S. O., Krutovskii, K. V. (1990). Species identity of the 'common mussel' inhabiting the Asiatic coasts of the Pacific Ocean. Biol. Morya, Vladivostok 1990(1): 12-22 (in Russian)

McDonald, J. H., Seed, R., Koehn, R. K. (1991). Allozymes and morphometric characters of three species of Mytilus in the northern and southern hemispheres. Mar Biol. 111. 323-333

Nei, M. (1972). Genetic distance between populations. Am Nat. 106: 283-292

Nei, M. (1977). F-statistics and analysis of gene diversity in subdivided populations. Ann. hum. Genet. 41. 225-233

Nei, M. (1986). Definition and estimation of fixation indices. Evolution 40: 643-645

Nei, M., Chesser, R. K. (1983). Estimation of fixation indices and gene diversities. Ann. hum. Genet. 47: 253-259

Nie, N. H., Hull, C. H., Jenkins, J. G., Steinbrenner, K., Bent, D. H. (1975). SPSS statistical package for the social sciences, 2nd edn. McGraw-Hill, New York

Pasteur, N., Pasteur, G., Bonhomme, F., Catalan, J., BrittonDavidian, J. (1987). Manuel technique de génétique par électrophorèse des protéines. Technique et documentation, Paris (English translation: Practical isozyme genetics. Ellis Horwood, Ltd, Chichester, 1988)

Pérès, J. M. (1967). Mediterranean benthos. Oceanogr. mar. Biol. A. Rev. 5: 449-533

Pérès, J. M. (1985). History of the Mediterranean biota and the colonization of the depths. In: Margalef, R. (ed.) Western Mediterranean. Key environment ser. Pergamon Press, Oxford, p. 198-232

Pimentel, R. A. (1979). Morphometrics. The multivariate analysis of biological data. Kendal/Hunt Publ. Co., Dubuque

Quesada, H., Sanjuan, J., Sanjuan, A. (1992). GENET2: a BASIC program for the analysis of deviations from HardyWeinberg Law. J. Heredity 83: 460-461

Raffi, S., Stanley, S. M., Marasti, R. (1985). Biogeographic patterns and Plio-pleistocene extinction of Bivalvia in the Mediterranean and southern North Sea. Paleobiology 11: $368-388$

Reyment, R. A., Blackith, R. E., Campbell, N. A. (1984). Multivariate morphometrics, 2nd edn. Academic Press, London

Robertson, A., Hill, W. G. (1984). Deviations from HardyWeinberg proportions, sampling variances and use in estimation of inbreeding coefficients. Genetics, Austin, Tex. 107: $703-718$

Rodriguez, J. (1982). Oceanografía del Mar Mediterráneo. Ediciones Pirámide, Madrid

Rohlf, F. J. (1990). NTSYS-pc. Numerical taxonomy and multivariate analysis system, ver. 1.50 edn. Exeter Software, New York

Ros, J. M., Romero, J., Ballesteros, E., Gili, J. M. (1985). Diving in blue water The benthos. In: Margalef, R. (ed.) Western Mediterranean. Key environment ser. Pergamon Press, Oxford, p. 233-295
Saavedra, C., Zapata, C., Guerra, A., Alvarez, G. (1993). Allozyme variation in European populations of the oyster Ostrea eduiis. Mar. Biol. 115: 85-95

Sanjuan, A. (1992). La identificación del mejillón de la Península lbérica: variabilidad morfológica y polimorfismos alozimicos. Tesis Doctoral, Univ. Santiago de Compostela

Sanjuan, A., Quesada, H., Zapata, C., Alvarez, G. (1986). Identificación del mejillón del NO. de la Península lbérica como Mytilus galloprovincialis Lmk. Acta Cient. Compostel. 23: $35-79$

Sanjuan, A., Quesada, H., Zapata, C., Alvarez, G. (1990). On the occurrence of Mytilus galloprovincialis Lmk. on the N.W. coast of the Iberian Peninsula. J. exp. mar. Biol. Ecol. 143: $1-14$

Sara, M. (1985). Ecological factors and their biogeographic consequences in the Mediterranean ecosystems In: Moraitou-Apostolopoulou, M., Kiortsis, V. (eds.) Mediterranean marine ecosystems. Plenum Press, London, p. 1-17

Saunders, N. C., Kessler, L. G., Avise, J. C. (1986). Genetic variation and geographic differentiation in mitochondrial DNA of the horsehoe crab, Limulus polyphemus. Genetics, Austin, Tex. 112: 613-627

Seed, R. (1972). Morphological variations in Mytilus from the French coasts in relation to the occurrence and distribution of M. galloprovincialis Lmk. Cah. Biol. mar. 13: 357-384

Seed, R. (1974). Morphological variation in Mytilus from Irish coasts in relation to the accurrence and distribution of Mytilus galloprovincialis Lmk. Cah. Biol. mar. 15: 1-25

Seed, R. (1976). Ecology. In: Bayne, B. L. (ed.) Marine mussels, their ecology and physiology. Cambridge Univ. Press, Cambridge, p. 13-65

Seed, R. (1978). The systematics and evolution of Mytilus galloprovincialis Lmk. In: Battaglia, B., Beardmore, J. A. (eds.) Marine organisms, genetics, ecology and evolution. Plenum Press, London, p. 447-468

Seed, R. (1992). Systematics, evolution, and distribution of mussels belonging to the genus Mytilus: an overview. Am. malacolog. Bull. 9: 123-137

Selander, R. K., Yang, S. Y., Lewontin, R. C., Johnson, W. C. (1970). Genetic variation in the horseshoe crab (Limulus polyphemus), a phylogenetic 'relic'. Evolution 24: 402-414

Skibinski, D. O. F. (1983). Natural selection in hybrid mussel populations. In: Oxford, G. S., Rollinson, D. (eds.) Systematics association special vol. 24. Protein polymorphism: adaptive and taxonomic significance. Academic Press, London, p. 283-298

Skibinski, D. O. F. (1985). Mitochondrial DNA variation in Mytilus edulis L. and the Padstow mussel. J. exp. mar. Biol. Ecol. 92: 251-258

Skibinski, D. O. F., Ahmad, M., Beardmore, J. A. (1978). Genetic evidence for naturally occurring hybrids between Mytilus edulis and Mytilus galloprovincialis. Evolution 32: $354-364$

Skibinski, D. O. F., Beardmore, J. A., Cross, T. F. (1983). Aspects of the population genetics of Mytilus (Mytilidae; Mollusca) in the British Isles. Biol. J. Linn. Soc. 19: 137-183

Skibinski, D. O. F., Cross, T F., Ahmad, M. (1980). Electrophoretic investigation of systematic relationships in the marine mussels Modiolus modiolus L., Mytilus edulis L. and Mytilus galloprovincialis Lmk (Mytilidae; Mollusca). Biol. J. Linn. Soc. 13: 65-73

Sneath, P. H. A., Sokal, R. R. (1973) Numerical taxonomy. Freeman, San Francisco

Suchanek, T H. (1985). Mussels and their rôle in structuring rocky shore communities. In: Moore, P. G., Seed, R. (eds.) The ecology of rocky shores. Hodder and Stoughton, London, p. 70-96 
Tintore, J., La Violette, P. E., Blade, I., Cruzado, G. (1988). A study of an intense density front in the eastern Alboran sea: the Almería-Oran front. $J$ phys. Oceanogr. 18: $1384-1397$

Varvio, S.-L., Koehn, R. K., Väinölä, R. (1988). Evolutionary genetics of the Mytilus edulis complex in the North Atlantic region. Mar. Biol. 98: 51-60

Verduin, A. (1979). Conchological evidence for the separate specific identity of Mytilus edulis L. and M. galloprovincialis Lam. Basteria 43;61-80

Vermeij, G. J. (1989). Invasion and extinction: the last three

This article was submitted to the editor million years of North Sea Pelecypod history. Conserv Biol. 3: 274-281

Ward, R. D. (1989). Molecular population genetics of marine organisms. In: Ryland, J. S., Tyler, P. A. (eds.) Reproduction, genetics and distributions of marine organisms. Proc 23rd Eur. mar. Biol. Symp. Olsen and Olsen, Fredensborg p. $235-249$

Willis, G. L., Skibinski, D.O.F. (1992) Variation in strengh of attachment to the sustrate explains differential mortality in hibrids mussels (Mytilus edulis and Mytilus galloprovincialis) populations. Mar. Biol. 112: 403-408

Manuscript first received: January 17, 1994

Revised version accepted: July 18, 1994 